\title{
2-track algebras and the Adams spectral sequence
}

\author{
Hans-Joachim Baues ${ }^{1}$ • Martin Frankland ${ }^{2}$
}

Received: 1 May 2015 / Accepted: 6 November 2015 / Published online: 24 November 2016

(C) Tbilisi Centre for Mathematical Sciences 2016

\begin{abstract}
In previous work of the first author and Jibladze, the $E_{3}$-term of the Adams spectral sequence was described as a secondary derived functor, defined via secondary chain complexes in a groupoid-enriched category. This led to computations of the $E_{3^{-}}$ term using the algebra of secondary cohomology operations. In work with Blanc, an analogous description was provided for all higher terms $E_{r}$. In this paper, we introduce 2-track algebras and tertiary chain complexes, and we show that the $E_{4}$-term of the Adams spectral sequence is a tertiary Ext group in this sense. This extends the work with Jibladze, while specializing the work with Blanc in a way that should be more amenable to computations.
\end{abstract}

Keywords Adams spectral sequence · Tertiary · Toda bracket · 2-track groupoid · Bigroupoid · Double groupoid

Mathematics Subject Classification 55T15 - 18G50 · 55S20

Dedicated to Ronnie Brown on the occasion of his eightieth birthday.

Communicated by Tim Porter and George Janelidze.

$凶 \quad$ Martin Frankland

martin.frankland@uni-osnabrueck.de

Hans-Joachim Baues

baues@mpim-bonn.mpg.de

1 Max-Planck-Institut für Mathematik, Vivatsgasse 7, 53111 Bonn, Germany

2 Institut für Mathematik, Universität Osnabrück, Albrechtstr. 28a, 49076 Osnabrück, Germany 


\section{Introduction}

A major problem in algebraic topology consists of computing homotopy classes of maps between spaces or spectra, notably the stable homotopy groups of spheres $\pi_{*}^{S}\left(S^{0}\right)$. One of the most useful tools for such computations is the Adams spectral sequence [1] (and its unstable analogues [8]), based on ordinary mod $p$ cohomology. Given finite spectra $X$ and $Y$, Adams constructed a spectral sequence of the form:

$$
E_{2}^{s, t}=\operatorname{Ext}_{\mathfrak{A}}^{s, t}\left(H^{*}\left(Y ; \mathbb{F}_{p}\right), H^{*}\left(X ; \mathbb{F}_{p}\right)\right) \Rightarrow\left[\Sigma^{t-s} X, Y_{p}^{\wedge}\right]
$$

where $\mathfrak{A}$ is the mod $p$ Steenrod algebra, consisting of primary stable mod $p$ cohomology operations, and $Y_{p}^{\wedge}$ denotes the $p$-completion of $Y$. In particular, taking sphere spectra $X=Y=S^{0}$, one obtains a spectral sequence

$$
E_{2}^{s, t}=\operatorname{Ext}_{\mathfrak{A}}^{s, t}\left(\mathbb{F}_{p}, \mathbb{F}_{p}\right) \Rightarrow \pi_{t-s}^{S}\left(S^{0}\right)_{p}^{\wedge}
$$

abutting to the $p$-completion of the stable homotopy groups of spheres. The differential $d_{r}$ is determined by $r$ th order cohomology operations [14]. In particular, secondary cohomology operations determine the differential $d_{2}$ and thus the $E_{3}$-term. The algebra of secondary operations was studied in [2]. In [3], the first author and Jibladze developed secondary chain complexes and secondary derived functors, and showed that the Adams $E_{3}$-term is given by secondary Ext groups of the secondary cohomology of $X$ and $Y$. They used this in [5], along with the algebra of secondary operations, to construct an algorithm that computes the differential $d_{2}$.

Primary operations in mod $p$ cohomology are encoded by the homotopy category $\operatorname{Ho}(\mathcal{K})$ of the Eilenberg-MacLane mapping theory $\mathcal{K}$, consisting of finite products of Eilenberg-MacLane spectra of the form $\Sigma^{n_{1}} H \mathbb{F}_{p} \times \cdots \times \Sigma^{n_{k}} H \mathbb{F}_{p}$. More generally, the $n$th Postnikov truncation $P_{n} \mathcal{K}$ of the Eilenberg-MacLane mapping theory encodes operations of order up to $n+1$, which in turn determine the Adams differential $d_{n+1}$ and thus the $E_{n+2}$-term [4]. However, $P_{n} \mathcal{K}$ contains too much information for practical purposes. In [6], the first author and Blanc extracted from $P_{n} \mathcal{K}$ the information needed in order to compute the Adams differential $d_{n+1}$. The resulting algebraic-combinatorial structure is called an algebra of left $n$-cubical balls.

In this paper, we specialize the work of [6] to the case $n=2$. Our goal is to provide an alternate structure which encodes an algebra of left 2-cubical balls, but which is more algebraic in nature and better suited for computations. The combinatorial difficulties in an algebra of left $n$-cubical balls arise from triangulations of the sphere $S^{n-1}=\partial D^{n}$. In the special case $n=2$, triangulations of the circle $S^{1}$ are easily described, unlike in the case $n>2$. Our approach also extends the work in [3] from secondary chain complexes to tertiary chain complexes.

\section{Organization and main results}

We define the notion of 2-track algebra (Definition 5.1) and show that each 2-track algebra naturally determines an algebra of left 2-cubical balls (Theorem 9.3). Build- 
ing on [6], we show that higher order resolutions always exist in a 2-track algebra (Theorem 8.7). We show that a suitable 2-track algebra related to the EilenbergMacLane mapping theory recovers the Adams spectral sequence up to the $E_{4}$-term (Theorem 7.3). We show that the spectral sequence only depends on the weak equivalence class of the 2-track algebra (Theorem 7.5).

Remark 1.1 This last point is important in view of the strictification result for secondary cohomology operations: these can be encoded by a graded pair algebra $B_{*}$ over $\mathbb{Z} / p^{2}[2, \S 5.5]$. The secondary Ext groups of the $E_{3}$-term turn out to be the usual Ext groups over $B_{*}$ [5, Theorem 3.1.1], a key fact for computations. We conjecture that a similar strictification result holds for tertiary operations, i.e., in the case $n=2$.

Appendix A explains why 2-track groupoids are not models for homotopy 2-types, and how to extract the underlying 2-track groupoid from a bigroupoid or a double groupoid.

\section{Cubes and tracks in a space}

In this section, we fix some notation and terminology regarding cubes and groupoids.

Definition 2.1 Let $X$ be a topological space.

An $n$-cube in $X$ is a map $a: I^{n} \rightarrow X$, where $I=[0,1]$ is the unit interval. For example, a 0 -cube in $X$ is a point of $X$, and a 1-cube in $X$ is a path in $X$.

An $n$-cube can be restricted to $(n-1)$-cubes along the $2 n$ faces of $I^{n}$. For $1 \leq i \leq n$, denote:

$$
\begin{aligned}
& d_{i}^{0}(a)=a \text { restricted to } I \times I \times \cdots \times \overbrace{\{0\}}^{i} \times \cdots \times I \\
& d_{i}^{1}(a)=a \text { restricted to } I \times I \times \cdots \times \overbrace{\{1\}}^{i} \times \cdots \times I .
\end{aligned}
$$

An $n$-track in $X$ is a homotopy class, relative to the boundary $\partial I^{n}$, of an $n$-cube. If $a: I^{n} \rightarrow X$ is an $n$-cube in $X$, denote by $\{a\}$ the corresponding $n$-track in $X$, namely the homotopy class of $a$ rel $\partial I^{n}$.

In particular, for $n=1$, a 1-track $\{a\}$ is a path homotopy class, i.e., a morphism in the fundamental groupoid of $X$ from $a(0)$ to $a(1)$. Let us fix our notation regarding groupoids. In this paper, we consider only small groupoids.

Notation 2.2 A groupoid is a (small) category in which every morphism is invertible. Denote the data of a groupoid by $G=\left(G_{0}, G_{1}, \delta_{0}, \delta_{1}\right.$, id $\left.^{\square}, \square,(-)^{\boxminus}\right)$, where:

- $G_{0}=\mathrm{Ob}(G)$ is the set of objects of $G$.

$-G_{1}=\operatorname{Hom}(G)$ is the set of morphisms of $G$. The set of morphisms from $x$ to $y$ is denoted $G(x, y)$. We write $x \in G$ and $\operatorname{deg}(x)=0$ for $x \in G_{0}$, and $\operatorname{deg}(x)=1$ for $x \in G_{1}$.

$-\delta_{0}: G_{1} \rightarrow G_{0}$ is the source map. 
$-\delta_{1}: G_{1} \rightarrow G_{0}$ is the target map.

- id $\mathrm{id}^{\square}: G_{0} \rightarrow G_{1}$ sends each object $x$ to its corresponding identity morphism id $\mathrm{id}_{x}^{\square}$.

- $\square: G_{1} \times G_{0} G_{1} \rightarrow G_{1}$ is composition in $G$.

$-f^{\boxminus}: y \rightarrow x$ is the inverse of the morphism $f: x \rightarrow y$.

Groupoids form a category $\mathbf{G p d}$, where morphisms are functors between groupoids.

For any object $x \in G_{0}$, denote by $\operatorname{Aut}_{G}(x)=G(x, x)$ the automorphism group of $x$.

Denote by $\operatorname{Comp}(G)=\pi_{0}(G)$ the components of $G$, i.e., the set of isomorphism classes of objects $G_{0} / \sim$.

Denote the fundamental groupoid of a topological space $X$ by $\Pi_{(1)}(X)$.

Definition 2.3 Let $X$ be a pointed space, with basepoint $0 \in X$. The constant map $0: I^{n} \rightarrow X$ with value $0 \in X$ is called the trivial $n$-cube.

A left 1-cube or left path in $X$ is a map $a: I \rightarrow X$ satisfying $a(1)=0$, that is, $d_{1}^{1}(a)=0$, the trivial 0 -cube. In other words, $a$ is a path in $X$ from a point $a(0)$ to the basepoint 0 . We denote $\delta a=a(0)$.

A left 2-cube in $X$ is a map $\alpha: I^{2} \rightarrow X$ satisfying $\alpha(1, t)=\alpha(t, 1)=0$ for all $t \in I$, that is, $d_{1}^{1}(\alpha)=d_{2}^{1}(\alpha)=0$, the trivial 1-cube.

More generally, a left $n$-cube in $X$ is a map $\alpha: I^{n} \rightarrow X$ satisfying $\alpha\left(t_{1}, \ldots, t_{n}\right)=0$ whenever some coordinate satisfies $t_{i}=1$. In other words, for all $1 \leq i \leq n$ we have $d_{i}^{1}(\alpha)=0$, the trivial $(n-1)$-cube.

A left $n$-track in $X$ is a homotopy class, relative to the boundary $\partial I^{n}$, of a left $n$-cube.

The equality $I^{m+n}=I^{m} \times I^{n}$ allows us to define an operation on cubes.

Definition 2.4 Let $\mu: X \times X^{\prime} \rightarrow X^{\prime \prime}$ be a map, for example a composition map in a topologically enriched category $\mathcal{C}$. For $m, n \geq 0$, consider cubes

$$
\begin{aligned}
& a: I^{m} \rightarrow X \\
& b: I^{n} \rightarrow X^{\prime} .
\end{aligned}
$$

The $\otimes$-composition of $a$ and $b$ is the $(m+n)$-cube $a \otimes b$ defined as the composite

$$
a \otimes b: I^{m+n}=I^{m} \times I^{n} \stackrel{a \times b}{\longrightarrow} X \times X^{\prime} \stackrel{\mu}{\rightarrow} X^{\prime \prime} .
$$

For $m=n$, the pointwise composition of $a$ and $b$ is the $n$-cube defined as the composite

$$
a b: I^{n} \stackrel{(a, b)}{\longrightarrow} X \times X^{\prime} \stackrel{\mu}{\rightarrow} X^{\prime \prime}
$$

The pointwise composition is the restriction of the $\otimes$-composition along the diagonal:

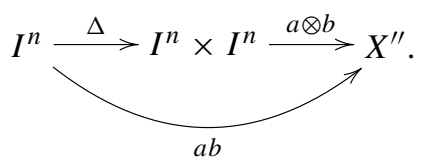


Remark 2.5 For $m=n=0$, the 0-cube $x \otimes y=x y$ is the pointwise composition, which is the composition in the underlying category. For higher dimensions, there are still relations between the $\otimes$-composition and the pointwise composition. In suggestive formulas, pointwise composition of paths is given by $(a b)(t)=a(t) b(t)$ for all $t \in I$, whereas the $\otimes$-composition of paths is the 2-cube given by $(a \otimes b)(s, t)=a(s) b(t)$.

Assume moreover that $\mu$ satisfies

$$
\mu(x, 0)=\mu\left(0, x^{\prime}\right)=0
$$

for the basepoints $0 \in X, 0 \in X^{\prime}, 0 \in X^{\prime \prime}$. For example, $\mu$ could be the composition map in a category $\mathcal{C}$ enriched in ( $\mathbf{T o p}_{*}, \wedge$ ), the category of pointed topological spaces with the smash product as monoidal structure. If $a$ and $b$ are left cubes, then $a \otimes b$ and $a b$ are also left cubes.

\section{2-track groupoids}

We now focus on left 2-tracks in a pointed space $X$, and observe that they form a groupoid. Define the groupoid $\Pi_{(2)}(X)$ with object set:

$$
\Pi_{(2)}(X)_{0}=\text { set of left } 1 \text {-cubes in } X
$$

and morphism set:

$$
\Pi_{(2)}(X)_{1}=\text { set of left 2-tracks in } X
$$

where the source $\delta_{0}$ and target $\delta_{1}$ of a left 2-track $\alpha: I \times I \rightarrow X$ are given by restrictions

$$
\begin{aligned}
& \delta_{0}(\alpha)=d_{1}^{0}(\alpha) \\
& \delta_{1}(\alpha)=d_{2}^{0}(\alpha)
\end{aligned}
$$

and note in particular $\delta \delta_{0}(\alpha)=\delta \delta_{1}(\alpha)=\alpha(0,0)$. In other words, a morphism $\alpha$ from $a$ to $b$ looks like this:

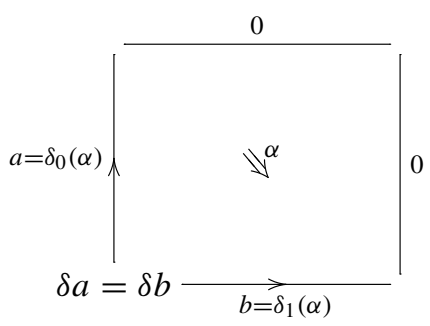


Remark 3.1 Up to reparametrization, a left 2-track $\alpha: a \Rightarrow b$ corresponds to a path homotopy from $a$ to $b$, which can be visualized in a globular picture:

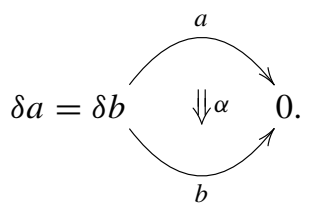

However, the $\otimes$-composition will play an important role in this paper, which is why we adopt a cubical approach, rather than globular or simplicial.

Composition $\beta \square \alpha$ of left 2-tracks is described by the following picture:

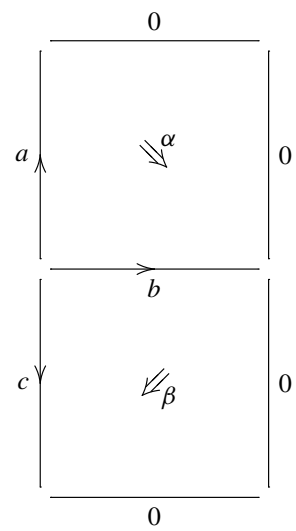

Remark 3.2 To make this definition precise, let $\alpha: a \Rightarrow b$ and $\beta: b \Rightarrow c$ be left 2-tracks in $X$, i.e., composable morphisms in $\Pi_{(2)}(X)$. Choose representative maps $\widetilde{\alpha}, \widetilde{\beta}: I^{2} \rightarrow X$. Consider the map $f_{\alpha, \beta}:[0,1] \times[-1,1] \rightarrow X$ pictured in (3.1). That is, define

$$
f(s, t)= \begin{cases}\widetilde{\alpha}(s, t) & \text { if } 0 \leq t \leq 1 \\ \widetilde{\beta}(-t, s) & \text { if }-1 \leq t \leq 0\end{cases}
$$

Now consider the reparametrization map $w: I^{2} \rightarrow[0,1] \times[-1,1]$ illustrated in this picture: 

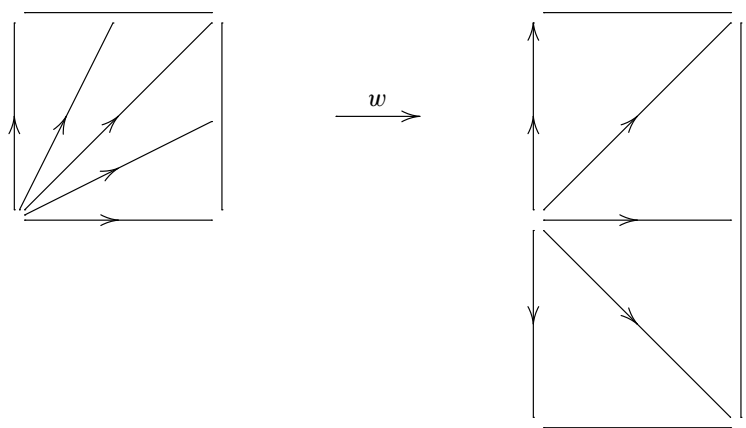

Explicitly, the restriction $\left.w\right|_{\partial I^{2}}$ to the boundary is the piecewise linear map satisfying

$$
\left\{\begin{array}{l}
w(0,0)=(0,0) \\
w(0,1)=(0,1) \\
w\left(\frac{1}{2}, 1\right)=(1,1) \\
w(1,1)=(1,0) \\
w\left(1, \frac{1}{2}\right)=(1,-1) \\
w(1,0)=(0,-1)
\end{array}\right.
$$

and $w(x)$ is defined for points $x \in I^{2}$ in the interior as follows. Write $x=p(0,0)+q y$ as a unique convex combination of $(0,0)$ and a point $y$ on the boundary $\partial I^{2}$. Then define $w(x)=p w(0,0)+q w(y)=q w(y)$. Finally, the composition $\beta \square \alpha: a \Rightarrow c$ is $\left\{f_{\alpha, \beta} \circ w\right\}$, the homotopy class of the composite

$$
I^{2} \stackrel{w}{\longrightarrow}[0,1] \times[-1,1] \stackrel{f_{\alpha, \beta}}{\longrightarrow} X
$$

relative to the boundary $\partial I^{2}$.

In other notation, we have inclusions $d_{2}^{0}: I^{1} \hookrightarrow I^{2}$ as the bottom edge $I \times\{0\}$ and $d_{1}^{0}: I^{1} \hookrightarrow I^{2}$ as the left edge $\{0\} \times I$, our $w$ is a map $w: I^{2} \rightarrow I^{2} \cup_{I^{1}} I^{2}$, and $\beta \square \alpha$ is the homotopy class of the composite

$$
I^{2} \stackrel{w}{\longrightarrow} I^{2} \cup_{I^{1}} I^{2} \stackrel{[\alpha \beta]}{\longrightarrow} X .
$$

Given a left path $a$ in $X$, the identity of $a$ in the groupoid $\Pi_{(2)}(X)$ is the left 2-track is pictured here: 


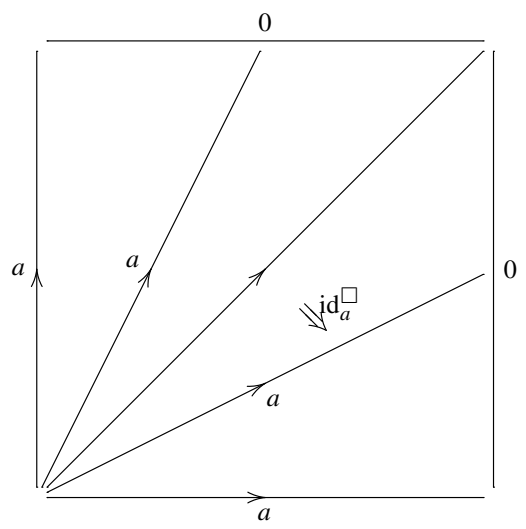

More precisely, for points $x \in I^{2}$ in the interior, write $x=p(0,0)+q y$ as a unique convex combination of $(0,0)$ and a point $y$ on the boundary $\partial I^{2}$. Then define $\operatorname{id}_{a}^{\square}(x)=$ $a(q)$.

The inverse $\alpha^{\boxminus:} b \Rightarrow a$ of a left 2-track $\alpha: a \Rightarrow b$ is the homotopy class of the composite $\alpha \circ T$, where $T: I^{2} \rightarrow I^{2}$ is the map swapping the two coordinates: $T(x, y)=(y, x)$.

Lemma 3.3 Given a pointed topological space $X$, the structure described above makes $\Pi_{(2)}(X)$ into a groupoid, called the groupoid of left 2 -tracks in X.

Proof Standard.

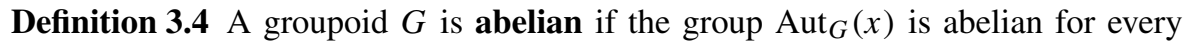
object $x \in G_{0}$. The groupoid $G$ is strictly abelian if it is pointed (with basepoint $0 \in G_{0}$ ), and is equipped with a family of isomorphisms

$$
\psi_{x}: \operatorname{Aut}_{G}(x) \stackrel{\simeq}{\rightarrow} \operatorname{Aut}_{G}(0)
$$

indexed by all objects $x \in G_{0}$, such that the diagram

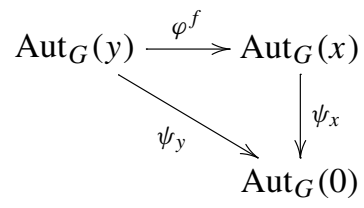

commutes for every map $f: x \rightarrow y$ in $G$, where $\varphi^{f}$ denotes the "change of basepoint" isomorphism

$$
\begin{aligned}
\varphi^{f}: \operatorname{Aut}_{G}(y) & \stackrel{\simeq}{\rightarrow} \operatorname{Aut}_{G}(x) \\
\alpha & \mapsto \varphi^{f}(\alpha)=f^{\boxminus} \square \alpha \square f .
\end{aligned}
$$


Remark 3.5 A strictly abelian groupoid is automatically abelian. Indeed, the compatibility condition (3.2) applied to automorphisms $f: 0 \rightarrow 0$ implies that conjugation $\varphi^{f}: \operatorname{Aut}_{G}(0) \rightarrow \operatorname{Aut}_{G}(0)$ is the identity.

Definition 3.6 A groupoid $G$ is pointed if it has a chosen basepoint, i.e., an object $0 \in G_{0}$. Here 0 is an abuse of notation: the basepoint is not assumed to be an initial object for $G$.

The star of a pointed groupoid $G$ is the set of all morphisms to the basepoint 0 , denoted by:

$$
\operatorname{Star}(G)=\left\{f \in G_{1} \mid \delta_{1}(f)=0\right\} .
$$

For a morphism $f: x \rightarrow 0$ in $\operatorname{Star}(G)$, we write $\delta f=\delta_{0} f=x$.

If $G$ has a basepoint $0 \in G_{0}$, then we take $\mathrm{id}_{0}^{\square} \in G_{1}$ as basepoint for the set of morphisms $G_{1}$ and for $\operatorname{Star}(G) \subseteq G_{1}$; we sometimes write $0=\mathrm{id}_{0}^{\square}$. Moreover, we take the component of the basepoint 0 as basepoint for $\operatorname{Comp}(G)$, the set of components of $G$.

Proposition 3.7 $\Pi_{(2)}(X)$ is a strictly abelian groupoid, and it satisfies Comp $\Pi_{(2)}(X)$ $\simeq \operatorname{Star} \Pi_{(1)}(X)$.

Proof Let $a \in \Pi_{(2)}(X)_{0}$ be a left path in $X$. To any automorphism $\alpha: 0 \Rightarrow 0$ in $\Pi_{(2)}(X)$, one can associate the well-defined left 2 -track indicated by the picture

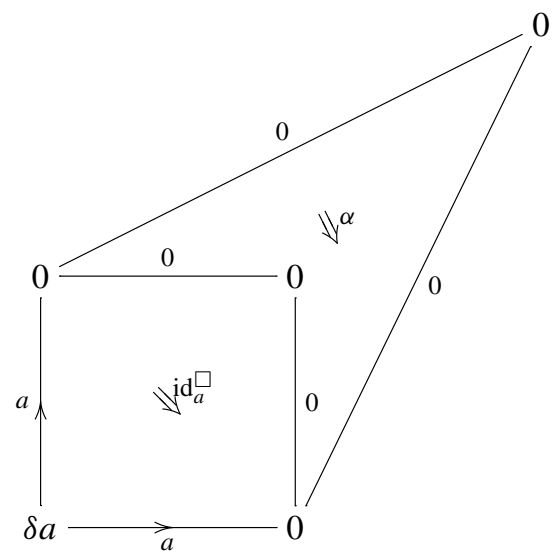

which is a morphism $a \Rightarrow a$. This assignment defines a map $\operatorname{Aut}_{\Pi_{(2)}(X)}(0) \rightarrow$ $\operatorname{Aut}_{\Pi_{(2)}(X)}(a)$ and is readily seen to be a group isomorphism, whose inverse we denote $\psi_{a}$. One readily checks that the family $\psi_{a}$ is compatible with change-of-basepoint isomorphisms.

The set Comp $\Pi_{(2)}(X)$ is the set of left paths in $X$ quotiented by the relation of being connected by a left 2-track. The set $\operatorname{Star} \Pi_{(1)}(X)$ is the set of left paths in $X$ quotiented by the relation of path homotopy. But two left paths are path-homotopic if and only if they are connected by a left 2-track. 
The bijection Comp $\Pi_{(2)}(X) \simeq \operatorname{Star} \Pi_{(1)}(X)$ is induced by taking the homotopy class of left 1-cubes. Consider the function $q: \Pi_{(2)}(X)_{0} \rightarrow \Pi_{(1)}(X)_{1}$ which sends a left 1-cube to its left 1-track $q(a)=\{a\}$. Then the image of $q$ is $\operatorname{Star} \Pi_{(1)}(X) \subseteq$ $\Pi_{(1)}(X)_{1}$ and $q$ is constant on the components of $\Pi_{(2)}(X)_{0}$. We now introduce a definition based on those features of $\Pi_{(2)}(X)$.

Definition 3.8 A 2 -track groupoid $G=\left(G_{(1)}, G_{(2)}\right)$ consists of:

- Pointed groupoids $G_{(1)}$ and $G_{(2)}$, with $G_{(2)}$ strictly abelian.

- A pointed function $q: G_{(2) 0} \rightarrow$ Star $G_{(1)}$ which is constant on the components of $G_{(2)}$, and such that the induced function $q: \operatorname{Comp} G_{(2)} \stackrel{\simeq}{\rightarrow} \operatorname{Star} G_{(1)}$ is bijective.

We assign degrees to the following elements:

$$
\operatorname{deg}(x)= \begin{cases}0 & \text { if } x \in G_{(1) 0} \\ 1 & \text { if } x \in G_{(2) 0} \\ 2 & \text { if } x \in G_{(2) 1}\end{cases}
$$

and we write $x \in G$ in each case.

A morphism of 2 -track groupoids $F: G \rightarrow G^{\prime}$ consists of a pair of pointed functors

$$
\begin{aligned}
& F_{(1)}: G_{(1)} \rightarrow G_{(1)}^{\prime} \\
& F_{(2)}: G_{(2)} \rightarrow G_{(2)}^{\prime}
\end{aligned}
$$

satisfying the following two conditions.

1. (Structural isomorphisms) For every object $a \in G_{(2) 0}$, the diagram

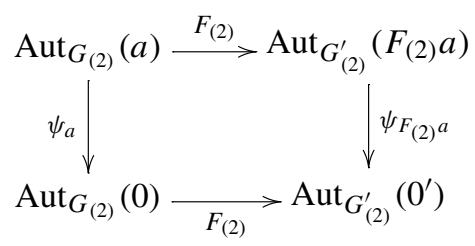

commutes.

2. (Quotient functions) The diagram

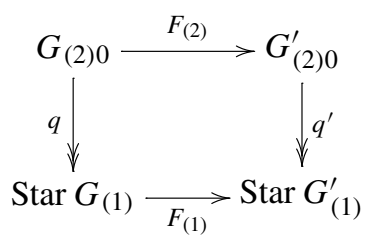

commutes. 
Let $\mathbf{G p d}_{(\mathbf{1}, \mathbf{2})}$ denote the category of 2-track groupoids.

Remark 3.9 If $\alpha: a \Rightarrow b$ is a left 2-track in a space, then the left paths $a$ and $b$ have the same starting point $\delta a=\delta b$. This condition is encoded in the definition of 2-track groupoid. Indeed, if $\alpha: a \Rightarrow b$ is a morphism in $G_{(2)}$, then $a, b \in G_{(2) 0}$ belong to the same component of $G_{(2)}$. Thus, we have $q(a)=q(b) \in \operatorname{Star} G_{(1)}$ and in particular $\delta q(a)=\delta q(b) \in G_{(1) 0}$.

Definition 3.10 The fundamental 2-track groupoid of a pointed space $X$ is

$$
\Pi_{(1,2)}(X):=\left(\Pi_{(1)}(X), \Pi_{(2)}(X)\right) .
$$

This construction defines a functor $\Pi_{(1,2)}: \mathbf{T o p}_{*} \rightarrow \mathbf{G p d}_{(\mathbf{1}, \mathbf{2})}$.

Remark 3.11 The grading on $\Pi_{(1,2)}(X)$ defined in Definition 3.8 corresponds to the dimension of the cubes. For $x \in \Pi_{(1,2)}(X)$, we have $\operatorname{deg}(x)=0$ if $x$ is a point in $X$, $\operatorname{deg}(x)=1$ if $x$ is a left path in $X$, and $\operatorname{deg}(x)=2$ if $x$ is a left 2-track in $X$. This 2-graded set is the left 2-cubical set $\mathrm{Nul}_{2}(X)$ [6, Definition 1.9].

Definition 3.12 Given a 2-track groupoid $G$, its homotopy groups are

$$
\begin{aligned}
\pi_{0} G & =\operatorname{Comp} G_{(1)} \\
\pi_{1} G & =\operatorname{Aut}_{G_{(1)}}(0) \\
\pi_{2} G & =\operatorname{Aut}_{G_{(2)}}(0) .
\end{aligned}
$$

Note that $\pi_{0} G$ is a priori only a pointed set, $\pi_{1} G$ is a group, and $\pi_{2} G$ is an abelian group.

A morphism $F: G \rightarrow G^{\prime}$ of 2-track groupoids is a weak equivalence if it induces an isomorphism on homotopy groups.

Remark 3.13 Let $X$ be a topological space with basepoint $x_{0} \in X$. Then the homotopy groups of its fundamental 2-track groupoid $G=\Pi_{(1,2)}\left(X, x_{0}\right)$ are the homotopy groups of the space $\pi_{i} G=\pi_{i}\left(X, x_{0}\right)$ for $i=0,1,2$.

The following two lemmas are straightforward.

Lemma 3.14 $\mathbf{G p d}_{(\mathbf{1 , 2})}$ has products, given by $G \times G^{\prime}=\left(G_{(1)} \times G_{(1)}^{\prime}, G_{(2)} \times G_{(2)}^{\prime}\right)$, and where the structural isomorphisms

$$
\psi_{\left(x, x^{\prime}\right)}: \operatorname{Aut}_{G_{(2)} \times G_{(2)}^{\prime}}\left(\left(x, x^{\prime}\right)\right) \stackrel{\simeq}{\rightarrow} \operatorname{Aut}_{G_{(2)} \times G_{(2)}^{\prime}}\left(\left(0,0^{\prime}\right)\right)
$$

are given by $\psi_{x} \times \psi_{x^{\prime}}$, and the quotient function

$$
\begin{aligned}
& \left(G \times G^{\prime}\right)_{(2) 0}=G_{(2) 0} \times G_{(2) 0}^{\prime} \\
& \Downarrow^{q \times q^{\prime}} \\
& \operatorname{Star}\left(G \times G^{\prime}\right)_{(1)}=\operatorname{Star} G_{(1)} \times \operatorname{Star} G_{(1)}^{\prime}
\end{aligned}
$$


is the product of the quotient functions for $G$ and $G^{\prime}$.

Lemma 3.15 The fundamental 2-track groupoid preserves products:

$$
\Pi_{(1,2)}(X \times Y) \cong \Pi_{(1,2)}(X) \times \Pi_{(1,2)}(Y)
$$

\section{2-tracks in a topologically enriched category}

Throughout this section, let $\mathcal{C}$ be a category enriched in $\left(\mathbf{T o p}_{*}, \wedge\right)$. Explicitly:

- For any objects $A$ and $B$ of $\mathcal{C}$, there is a morphism space $\mathcal{C}(A, B)$ with basepoint denoted $0 \in \mathcal{C}(A, B)$.

- For any objects $A, B$, and $C$, there is a composition map

$$
\mu: \mathcal{C}(B, C) \times \mathcal{C}(A, B) \rightarrow \mathcal{C}(A, C)
$$

which is associative and unital.

- Composition satisfies

$$
\mu(x, 0)=\mu(0, y)=0
$$

for all $x$ and $y$.

We write $x \in \mathcal{C}$ if $x \in \mathcal{C}(A, B)$ for some objects $A$ and $B$. For $x, y \in \mathcal{C}$, we write $x y=\mu(x, y)$ when $x$ and $y$ are composable, i.e., when the target of $y$ is the source of $x$. From now on, whenever an expression such as $x y$ or $x \otimes y$ appears, it is understood that $x$ and $y$ must be composable.

By Definition 2.4, we have the $\otimes$-composition $x \otimes y$ for $x, y \in \Pi_{(1)} \mathcal{C}$ and $\operatorname{deg}(x)+$ $\operatorname{deg}(y) \leq 1$. For $\operatorname{deg}(a)=\operatorname{deg}(b)=1$, we have:

$$
\begin{aligned}
a b & =\left(a \otimes \delta_{1} b\right) \square\left(\delta_{0} a \otimes b\right) \\
& =\left(\delta_{1} a \otimes b\right) \square\left(a \otimes \delta_{0} b\right)
\end{aligned}
$$

This equation holds in any category enriched in groupoids, where $a b$ denotes the (pointwise) composition. Note that for paths $\widetilde{a}$ and $\widetilde{b}$ representing $a$ and $b$, the boundary of the 2-cube $\widetilde{a} \otimes \widetilde{b}$ corresponds to the equation.

Conversely, the $\otimes$-composition in $\Pi_{(1)} \mathcal{C}$ is determined by the pointwise composition. For $\operatorname{deg}(x)=\operatorname{deg}(y)=0$ and $\operatorname{deg}(a)=1$, we have:

$$
\left\{\begin{array}{l}
x \otimes y=x y \\
x \otimes a=\mathrm{id}_{x} a \\
a \otimes x=a \mathrm{id}_{x}^{\square} .
\end{array}\right.
$$

We now consider the 2-track groupoids $\Pi_{(1,2)} \mathcal{C}(A, B)$ of morphism spaces in $\mathcal{C}$, and we write $x \in \Pi_{(1,2)} \mathcal{C}$ if $x \in \Pi_{(1,2)} \mathcal{C}(A, B)$ for some objects $A, B$ of $\mathcal{C}$. By Definition 2.4, composition in $\mathcal{C}$ induces the $\otimes$-composition: 


$$
x \otimes y \in \Pi_{(1,2)} \mathcal{C}
$$

if $x$ and $y$ satisfy $\operatorname{deg}(x)+\operatorname{deg}(y) \leq 2$. For $\operatorname{deg}(x)=\operatorname{deg}(y)=1, x$ and $y$ are left paths, hence $x \otimes y$ is well-defined. The $\otimes$-composition satisfies:

$$
\operatorname{deg}(x \otimes y)=\operatorname{deg}(x)+\operatorname{deg}(y)
$$

The $\otimes$-composition is associative, since composition in $\mathcal{C}$ is associative. The identity elements $1_{A} \in \mathcal{C}(A, A)$ for $\mathcal{C}$ provide identity elements $1=1_{A} \in \Pi_{(1,2)} \mathcal{C}(A, A)$, with $\operatorname{deg}\left(1_{A}\right)=0$, and $x \otimes 1=x=1 \otimes x$.

Let us describe the $\otimes$-composition of left paths more explicitly. Given left paths $a$ and $b$, then $a \otimes b$ is a 2-track from $\delta_{0}(a \otimes b)=(\delta a) \otimes b$ to $\delta_{1}(a \otimes b)=a \otimes(\delta b)$, as illustrated here:

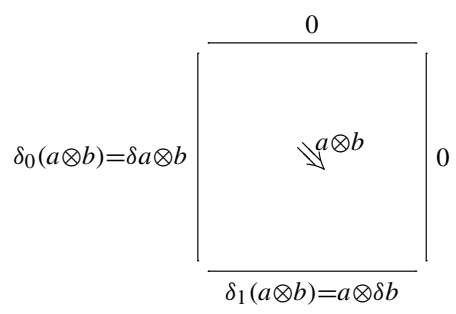

Definition 4.1 The 2 -track algebra associated to $\mathcal{C}$, denoted $\left(\Pi_{(1)} \mathcal{C}, \Pi_{(1,2)} \mathcal{C}, \square, \otimes\right)$, consists of the following data.

- $\Pi_{(1)} \mathcal{C}$ is the category enriched in pointed groupoids given by the fundamental groupoids $\left(\Pi_{(1)} \mathcal{C}(A, B), \square\right)$ of morphism spaces in $\mathcal{C}$, along with the $\otimes$ composition, which determines (and is determined by) the composition in $\Pi_{(1)} \mathcal{C}$.

- $\Pi_{(1,2)} \mathcal{C}$ is given by the collection of fundamental 2 -track groupoids $\left(\Pi_{(1,2)} \mathcal{C}(A, B)\right.$, $\square)$ together with the $\otimes$-composition $x \otimes y$ for $x, y \in \Pi_{(1,2)} \mathcal{C}$ satisfying $\operatorname{deg}(x)+\operatorname{deg}(y) \leq 2$.

Proposition 4.2 Let $x, \alpha, \beta \in \Pi_{(1,2)} \mathcal{C}$ with $\operatorname{deg}(x)=0$ and $\operatorname{deg}(\alpha)=\operatorname{deg}(\beta)=2$. Then the following equations hold:

$$
\left\{\begin{array}{l}
x \otimes(\beta \square \alpha)=(x \otimes \beta) \square(x \otimes \alpha) \\
(\beta \square \alpha) \otimes x=(\beta \otimes x) \square(\alpha \otimes x) .
\end{array}\right.
$$

Proof This follows from functoriality of $\Pi_{(2)}$ applied to the composition maps $\mu(x,-): \mathcal{C}(A, B) \rightarrow \mathcal{C}(A, C)$ and $\mu(-, x): \mathcal{C}(B, C) \rightarrow \mathcal{C}(A, C)$.

Proposition 4.3 Let $c, \alpha \in \Pi_{(1,2)} \mathcal{C}$ with $\operatorname{deg}(c)=1$ and $\operatorname{deg}(\alpha)=2$. Then the following equations hold:

$$
\left\{\begin{array}{l}
\delta_{1} \alpha \otimes c=(\alpha \otimes \delta c) \square\left(\delta_{0} \alpha \otimes c\right) \\
c \otimes \delta_{0} \alpha=\left(c \otimes \delta_{1} \alpha\right) \square(\delta c \otimes \alpha) .
\end{array}\right.
$$


Proof Write $a=\delta_{0} \alpha$ and $b=\delta_{1} \alpha$, i.e., $\alpha$ is a left 2-track from $a$ to $b$ :

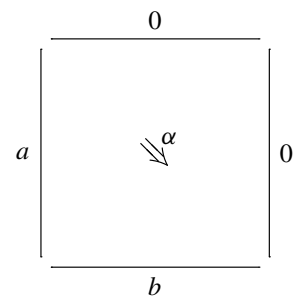

and note in particular $\delta a=\delta b$. Let $\widetilde{\alpha}$ be a left 2-cube that represents $\alpha$ and consider the left 3-cube $\widetilde{\alpha} \otimes c$ :

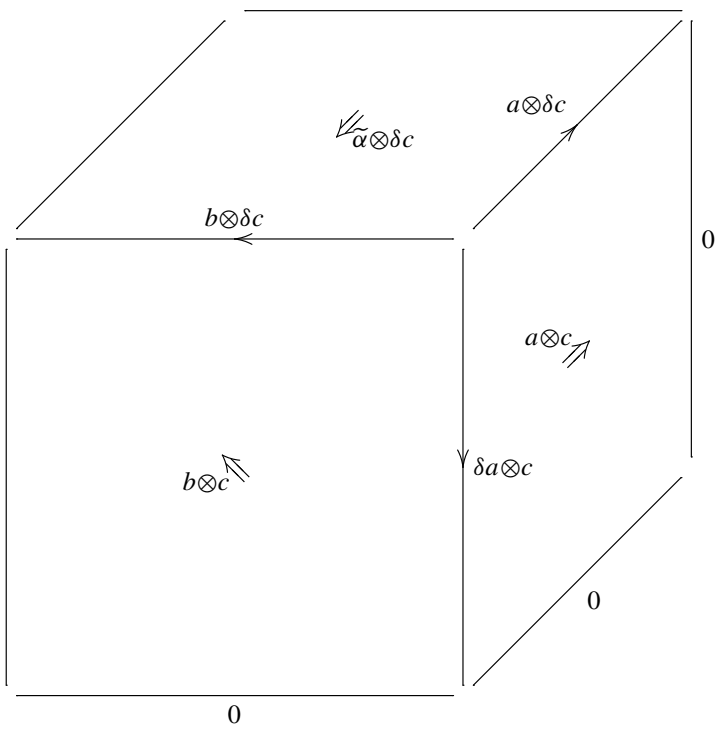

Its boundary exhibits the equality of 2-tracks:

$$
\begin{aligned}
& \text { top face } \square \text { right face }=\text { front face } \\
& (\alpha \otimes \delta c) \square(a \otimes c)=b \otimes c \\
& (\alpha \otimes \delta c) \square\left(\delta_{0} \alpha \otimes c\right)=\delta_{1} \alpha \otimes c .
\end{aligned}
$$

Likewise, for the second equation, consider the left 3-cube $c \otimes \widetilde{\alpha}$ : 


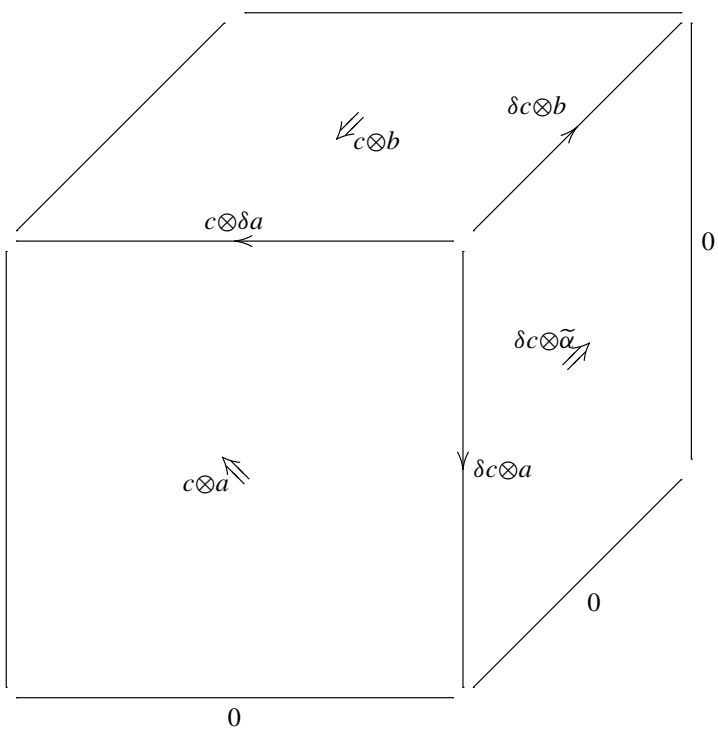

Its boundary exhibits the equality of 2-tracks:

$$
\begin{aligned}
& \text { top face } \square \text { right face }=\text { front face } \\
& (c \otimes b) \square(\delta c \otimes \alpha)=c \otimes a \\
& \left(c \otimes \delta_{1} \alpha\right) \square(\delta c \otimes \alpha)=c \otimes \delta_{0} \alpha .
\end{aligned}
$$

\section{2-track algebras}

We now collect the structure found in $\left(\Pi_{(1)} \mathcal{C}, \Pi_{(1,2)} \mathcal{C}, \square, \otimes\right)$ into the following definition.

Definition 5.1 A 2 -track algebra $\mathcal{A}=\left(\mathcal{A}_{(1)}, \mathcal{A}_{(1,2)}, \square, \otimes\right)$ consists of the following data.

1 A category $\mathcal{A}_{(1)}$ enriched in pointed groupoids, with the $\otimes$-composition determined by Eq. (4.1).

2 A collection $\mathcal{A}_{(1,2)}$ of 2-track groupoids $\left(\mathcal{A}_{(1,2)}(A, B), \square\right)$ for all objects $A, B$ of $\mathcal{A}_{(1)}$, such that the first groupoid in $\mathcal{A}_{(1,2)}(A, B)$ is equal to the pointed groupoid $\mathcal{A}_{(1)}(A, B)$.

3 For $x, y \in \mathcal{A}_{(1,2)}$, the $\otimes$-composition $x \otimes y \in \mathcal{A}_{(1,2)}$ is defined. For $\operatorname{deg}(x)=0$ and $\operatorname{deg}(y)=1$, the following equations hold in $\mathcal{A}_{(1)}$ :

$$
\left\{\begin{array}{l}
q(x \otimes y)=x \otimes q(y) \\
q(y \otimes x)=q(y) \otimes x .
\end{array}\right.
$$


The following equations are required to hold.

1. (Associativity) $\otimes$ is associative: $(x \otimes y) \otimes z=x \otimes(y \otimes z)$.

2. (Units) The units $1 \in \mathcal{A}_{(1)}$, with $\operatorname{deg}\left(1_{A}\right)=0$, serve as units for $\otimes$, i.e., satisfy $x \otimes 1=x=1 \otimes x$ for all $x \in \mathcal{A}_{(1,2)}$.

3. (Pointedness) $\otimes$ satisfies $x \otimes 0=0$ and $0 \otimes y=0$.

4. For $x, y, \alpha, \beta \in \mathcal{A}_{(1,2)}$ with $\operatorname{deg}(x)=\operatorname{deg}(y)=0$ and $\operatorname{deg}(\alpha)=\operatorname{deg}(\beta)=2$, we have:

$$
\left\{\begin{array}{l}
\delta_{i}(x \otimes \alpha \otimes y)=x \otimes\left(\delta_{i} \alpha\right) \otimes y \quad \text { for } i=0,1 \\
x \otimes(\beta \square \alpha) \otimes y=(x \otimes \beta \otimes y) \square(x \otimes \alpha \otimes y)
\end{array}\right.
$$

5. For $a, b \in \mathcal{A}_{(1,2)}$ with $\operatorname{deg}(a)=\operatorname{deg}(b)=1$, we have:

$$
\left\{\begin{array}{l}
\delta_{0}(a \otimes b)=\delta a \otimes b \\
\delta_{1}(a \otimes b)=a \otimes \delta b .
\end{array}\right.
$$

6. For $c, \alpha \in \mathcal{A}_{(1,2)}$ with $\operatorname{deg}(c)=1$ and $\operatorname{deg}(\alpha)=2$, we have:

$$
\left\{\begin{array}{l}
\delta_{1} \alpha \otimes c=(\alpha \otimes \delta c) \square\left(\delta_{0} \alpha \otimes c\right) \\
c \otimes \delta_{0} \alpha=\left(c \otimes \delta_{1} \alpha\right) \square(\delta c \otimes \alpha) .
\end{array}\right.
$$

Definition 5.2 A morphism of 2 -track algebras $F: \mathcal{A} \rightarrow \mathcal{B}$ consists of the following.

1. A functor $F_{(1)}: \mathcal{A}_{(1)} \rightarrow \mathcal{B}_{(1)}$ enriched in pointed groupoids.

2. A collection $F_{(1,2)}$ of morphisms of 2-track groupoids

$$
F_{(1,2)}(A, B): \mathcal{A}_{(1,2)}(A, B) \rightarrow \mathcal{B}_{(1,2)}(F A, F B)
$$

for all objects $A, B$ of $\mathcal{A}$, such that $F_{(1,2)}(A, B)$ restricted to the first groupoid in $\mathcal{A}_{(1,2)}(A, B)$ is the functor $F_{(1)}(A, B): \mathcal{A}_{(1)}(A, B) \rightarrow \mathcal{B}_{(1)}(F A, F B)$.

3. (Compatibility with $\otimes) F$ commutes with $\otimes$ :

$$
F(x \otimes y)=F x \otimes F y .
$$

Denote by $\mathbf{A l g}(\mathbf{1}, \mathbf{2})$ the category of 2-track algebras.

Definition 5.3 Let $\mathcal{A}$ be a 2-track algebra. The underlying homotopy category of $\mathcal{A}$ is the homotopy category of the underlying track category $\mathcal{A}_{(1)}$, denoted

$$
\pi_{0} \mathcal{A}:=\pi_{0} \mathcal{A}_{(1)}=\operatorname{Comp} \mathcal{A}_{(1)} .
$$

We say that $\mathcal{A}$ is based on the category $\pi_{0} \mathcal{A}$.

Definition 5.4 A morphism of 2-track algebras $F: \mathcal{A} \rightarrow \mathcal{B}$ is a weak equivalence (or Dwyer-Kan equivalence) if the following conditions hold: 
1. For all objects $A$ and $B$ of $\mathcal{A}$, the morphism

$$
F_{(1,2)}: \mathcal{A}_{(1,2)}(A, B) \rightarrow \mathcal{B}_{(1,2)}(F A, F B)
$$

is a weak equivalence of 2-track groupoids (Definition 3.12).

2. The induced functor $\pi_{0} F: \pi_{0} \mathcal{A} \rightarrow \pi_{0} \mathcal{B}$ is an equivalence of categories.

\section{Higher order chain complexes}

In this section, we construct tertiary chain complexes, extending the work of [3] on secondary chain complexes. We will follow the treatment therein.

Definition 6.1 A chain complex $(A, d)$ in a pointed category $\mathbf{A}$ is a sequence of objects and morphisms

$$
\cdots \longrightarrow A_{n+1} \stackrel{d_{n}}{\longrightarrow} A_{n} \stackrel{d_{n-1}}{\longrightarrow} A_{n-1} \longrightarrow \cdots
$$

in A satisfying $d_{n-1} d_{n}=0$ for all $n \in \mathbb{Z}$. The map $d$ is called the differential.

A chain map $f:(A, d) \rightarrow\left(A^{\prime}, d^{\prime}\right)$ between chain complexes is a sequence of morphisms $f_{n}: A_{n} \rightarrow A_{n}^{\prime}$ commuting with the differentials:

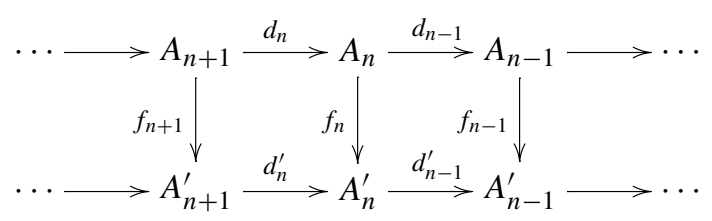

i.e., satisfying $f_{n} d_{n}=d_{n}^{\prime} f_{n+1}$ for all $n \in \mathbb{Z}$.

Definition 6.2 [3, Definition 2.6] Let $\mathbf{B}$ be a category enriched in pointed groupoids. A secondary pre-chain complex $(A, d, \gamma)$ in $\mathbf{B}$ is a diagram of the form:

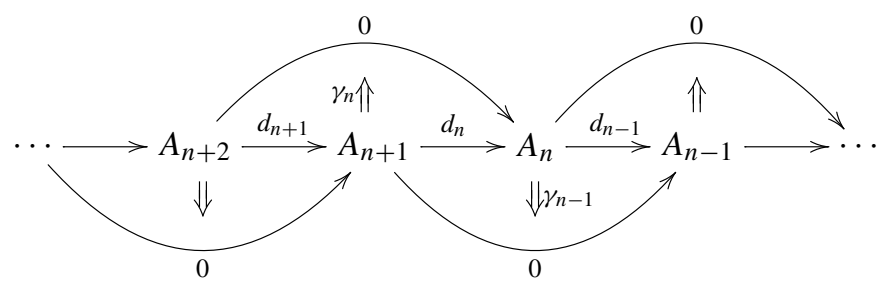

More precisely, the data consists of a sequence of objects $A_{n}$ and maps $d_{n}: A_{n+1} \rightarrow$ $A_{n}$, together with left tracks $\gamma_{n}: d_{n} d_{n+1} \Rightarrow 0$ for all $n \in \mathbb{Z}$. 
$(A, d, \gamma)$ is a secondary chain complex if moreover for each $n \in \mathbb{Z}$, the tracks

$$
d_{n-1} d_{n} d_{n+1} \stackrel{d_{n-1} \otimes \gamma_{n}}{\longrightarrow} d_{n-1} 0 \stackrel{\mathrm{id}_{0}^{\square}}{\Longrightarrow} 0
$$

and

$$
d_{n-1} d_{n} d_{n+1} \stackrel{\gamma_{n-1} \otimes d_{n+1}}{\Longrightarrow} 0 d_{n+1} \stackrel{\mathrm{id}_{0}^{\square}}{\Longrightarrow} 0
$$

coincide. In other words, the track

$$
\mathcal{O}\left(\gamma_{n-1}, \gamma_{n}\right):=\left(\gamma_{n-1} \otimes d_{n+1}\right) \square\left(d_{n-1} \otimes \gamma_{n}\right)^{\boxminus}: 0 \Rightarrow 0
$$

in the groupoid $\mathbf{B}\left(A_{n+2}, A_{n-1}\right)$ is the identity track of 0 .

We say that the secondary pre-chain complex $(A, d, \gamma)$ is based on the chain complex $(A,\{d\})$ in the homotopy category $\pi_{0} \mathbf{B}$.

Remark 6.3 One can show that the notion of secondary (pre-)chain complex in $\mathbf{B}$ coincides with the notion of $1^{\text {st }}$ order (pre-)chain complex in $\mathrm{Nul}_{1} \mathbf{B}$ described in $[6$, $\S 4$, c.f. Example 12.3].

Definition 6.4 A tertiary pre-chain complex $(A, d, \delta, \xi)$ in a 2-track algebra $\mathcal{A}$ is a sequence of objects $A_{n}$ and maps $d_{n}: A_{n+1} \rightarrow A_{n}$ in the category $\mathcal{A}_{(1) 0}$, together with left paths $\gamma_{n}: d_{n} d_{n+1} \rightarrow 0$ in $\mathcal{A}_{(1,2)}$, as illustrated in the diagram

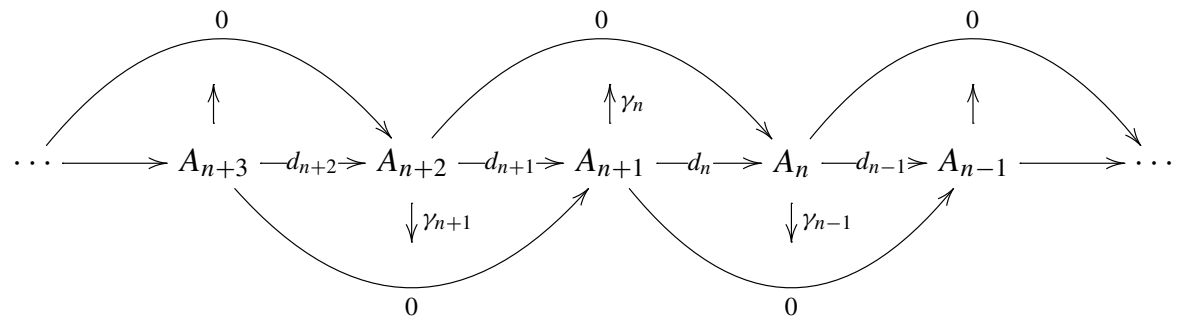

along with left 2-tracks $\xi_{n}: \gamma_{n} \otimes d_{n+2} \Rightarrow d_{n} \otimes \gamma_{n+1}$ in $\mathcal{A}_{(1,2)}$, for all $n \in \mathbb{Z}$.

$(A, d, \gamma, \xi)$ is a tertiary chain complex if moreover for each $n \in \mathbb{Z}$, the left 2-track:

$$
d_{n-1} \otimes \gamma_{n} \otimes d_{n+2} \stackrel{d_{n-1} \otimes \xi_{n}}{\longrightarrow} d_{n-1} d_{n} \otimes \gamma_{n+1} \stackrel{\gamma_{n-1} \otimes \gamma_{n+1}}{\Longrightarrow} \gamma_{n-1} \otimes d_{n+1} d_{n+2} \stackrel{\xi_{n-1} \otimes d_{n+2}}{\longrightarrow} d_{n-1} \otimes \gamma_{n} \otimes d_{n+2}
$$

is the identity of $d_{n-1} \otimes \gamma_{n} \otimes d_{n+2}$ in the groupoid $\mathcal{A}_{(2)}\left(A_{n+3}, A_{n-1}\right)$. In other words, the element:

$$
\begin{aligned}
\mathcal{O}\left(\xi_{n-1}, \xi_{n}\right) & :=\psi_{d_{n-1} \otimes \gamma_{n} \otimes d_{n+2}}\left(\left(\xi_{n-1} \otimes d_{n+2}\right) \square\left(\gamma_{n-1} \otimes \gamma_{n+1}\right) \square\left(d_{n-1} \otimes \xi_{n}\right)\right) \\
& \in \pi_{2} \mathcal{A}_{(1,2)}\left(A_{n+3}, A_{n-1}\right)
\end{aligned}
$$


is trivial. Here, $\psi$ is the structural isomorphism in the 2-track groupoid $\mathcal{A}_{(1,2)}\left(A_{n+3}\right.$, $A_{n-1}$ ), as in Definitions 3.4 and 3.8.

We say that the tertiary pre-chain complex $(A, d, \gamma, \xi)$ is based on the chain complex $(A,\{d\})$ in the homotopy category $\pi_{0} \mathcal{A}$.

\subsection{Toda brackets of length 3 and 4}

Let $\mathcal{C}$ be a category enriched in $\left(\mathbf{T o p}_{*}, \wedge\right)$. Let $\pi_{0} \mathcal{C}$ be the category of path components of $\mathcal{C}$ (applied to each mapping space) and let

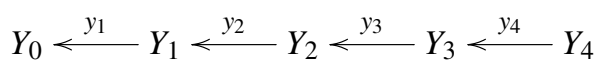

be a diagram in $\pi_{0} \mathcal{C}$ satisfying $y_{1} y_{2}=0, y_{2} y_{3}=0$, and $y_{3} y_{4}=0$. Choose maps $x_{i}$ in $\mathcal{C}$ representing $y_{i}$. Then there exist left 1-cubes $a, b, c$ as in the diagram

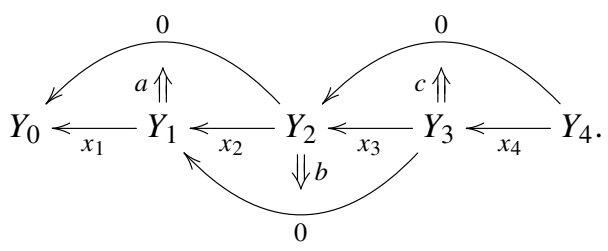

Definition 6.5 The Toda bracket of length 3 , denoted $\left\langle y_{1}, y_{2}, y_{3}\right\rangle \subseteq \pi_{1} \mathcal{C}\left(Y_{3}, Y_{0}\right)$, is the set of all elements in $\operatorname{Aut}(0)=\pi_{1} \mathcal{C}\left(Y_{3}, Y_{0}\right)$ of the form

$$
\mathcal{O}(a, b):=\left(a \otimes x_{3}\right) \square\left(x_{1} \otimes b\right)^{\boxminus}
$$

as above.

Assume now that we can choose left 2-tracks $\alpha: a \otimes x_{3} \Rightarrow x_{1} \otimes b$ and $\beta: b \otimes x_{4} \Rightarrow$ $x_{2} \otimes c$ in $\Pi_{(1,2)} \mathcal{C}$. Then the composite of left 2-tracks

$$
\left(\alpha \otimes x_{4}\right) \square(a \otimes c) \square\left(x_{1} \otimes \beta\right)
$$

is an element of $\operatorname{Aut}\left(x_{1} \otimes b \otimes x_{4}\right)$, to which we apply the structural isomorphism

$$
\psi_{x_{1} \otimes b \otimes x_{4}}: \operatorname{Aut}\left(x_{1} \otimes b \otimes x_{4}\right) \stackrel{\cong}{\rightarrow} \pi_{2} \mathcal{C}\left(Y_{4}, Y_{0}\right)
$$

The set of all such elements is the Toda bracket of length 4, denoted $\left\langle y_{1}, y_{2}, y_{3}, y_{4}\right\rangle \subseteq$ $\pi_{2} \mathcal{C}\left(Y_{4}, Y_{0}\right)$.

Note that the existence of $\alpha$, resp. $\beta$, implies that the bracket $\left\langle y_{1}, y_{2}, y_{3}\right\rangle$, resp. $\left\langle y_{2}, y_{3}, y_{4}\right\rangle$ contains the zero element. 
Remark 6.6 For a secondary pre-chain complex $(A, d, \gamma)$, we have

$$
\mathcal{O}\left(\gamma_{n-1}, \gamma_{n}\right) \in\left\langle d_{n-1}, d_{n}, d_{n+1}\right\rangle
$$

for every $n \in \mathbb{Z}$. Likewise, for a tertiary pre-chain complex $(A, d, \gamma, \xi)$, we have

$$
\mathcal{O}\left(\xi_{n-1}, \xi_{n}\right) \in\left\langle d_{n-1}, d_{n}, d_{n+1}, d_{n+2}\right\rangle
$$

for every $n \in \mathbb{Z}$. However, the vanishing of these Toda brackets does not guarantee the existence of a tertiary chain complex based on the chain complex $(A,\{d\})$. In a secondary chain complex $(A, d, \gamma)$, these Toda brackets vanish in a compatible way, that is, the equations $\mathcal{O}\left(\gamma_{n-1}, \gamma_{n}\right)=0$ and $\mathcal{O}\left(\gamma_{n}, \gamma_{n+1}\right)=0$ involve the same left track $\gamma_{n}: d_{n} d_{n+1} \Rightarrow 0$.

\section{The Adams differential $d_{3}$}

Let Spec denote the topologically enriched category of spectra and mapping spaces between them. More precisely, start from a simplicial (or topological) model category of spectra, like that of Bousfield-Friedlander [9, §2], or symmetric spectra or orthogonal spectra [13], and take Spec to be the full subcategory of fibrant-cofibrant objects; c.f. [6, Example 7.3].

Let $H:=H \mathbb{F}_{p}$ be the Eilenberg-MacLane spectrum for the prime $p$ and let $\mathfrak{A}=H^{*} H$ denote the mod $p$ Steenrod algebra. Consider the collection EM of all $\bmod p$ generalized Eilenberg-MacLane spectra that are bounded below and of finite type, i.e., degreewise finite products $A=\prod_{i} \Sigma^{n_{i}} H$ with $n_{i} \in \mathbb{Z}$ and $n_{i} \geq N$ for some integer $N$ for all $i$. Since the product is degreewise finite, the natural map $\bigvee_{i} \Sigma^{n_{i}} H \rightarrow \prod_{i} \Sigma^{n_{i}} H$ is an equivalence, so that the mod $p$ cohomology $H^{*} A$ is a free $\mathfrak{A}$-module. Moreover, the cohomology functor restricted to the full subcategory of Spec with objects EM yields an equivalence of categories in the diagram:

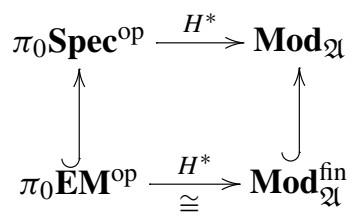

where $\operatorname{Mod}_{\mathfrak{A}}^{\text {fin }}$ denotes the full subcategory consisting of free $\mathfrak{A}$-modules which are bounded below and of finite type.

Given spectra $Y$ and $X$, consider the Adams spectral sequence:

$$
E_{2}^{s, t}=\operatorname{Ext}_{\mathfrak{A}}^{s, t}\left(H^{*} X, H^{*} Y\right) \Rightarrow\left[\Sigma^{t-s} Y, X_{p}^{\wedge}\right]
$$

Assume that $Y$ is a finite spectrum and $X$ is a connective spectrum of finite type, i.e., $X$ is equivalent to a $\mathrm{CW}$-spectrum with finitely many cells in each dimension and no cells 
below a certain dimension. Then the mod $p$ cohomology $H^{*} X$ is an $\mathfrak{A}$-module which is bounded below and degreewise finitely generated (as an $\mathfrak{A}$-module, or equivalently, as an $\mathbb{F}_{p}$-vector space). Choose a free resolution of $H^{*} X$ as an $\mathfrak{A}$-module:

$$
\cdots \longrightarrow F_{2} \stackrel{e_{1}}{\longrightarrow} F_{1} \stackrel{e_{0}}{\longrightarrow} F_{0} \stackrel{\lambda}{\longrightarrow} H^{*} X
$$

where each $F_{i}$ is a free $\mathfrak{A}$-module of finite type and bounded below. This diagram can be realized as the cohomology of a diagram in the stable homotopy category $\pi_{0}$ Spec:

$$
\cdots \longleftarrow A_{2} \longleftarrow d_{1} A_{1} \stackrel{d_{0}}{\longleftarrow} A_{0} \longleftarrow \epsilon \quad A_{-1}=X
$$

with each $A_{i}$ in $\mathbf{E M}$ (for $i \geq 0$ ) and satisfying $H^{*} A_{i} \cong F_{i}$. We consider this diagram as a diagram in the opposite category $\pi_{0}$ Spec $^{\text {op }}$ of the form:

$$
\cdots \longrightarrow A_{2} \stackrel{d_{1}}{\longrightarrow} A_{1} \stackrel{d_{0}}{\longrightarrow} A_{0} \stackrel{\epsilon}{\longrightarrow} A_{-1}=X
$$

Since $A_{\bullet} \rightarrow X$ is an EM-resolution of $X$ in $\pi_{0}$ Spec $^{\text {op }}$, there exists a tertiary chain complex $(A, d, \gamma, \xi)$ in $\Pi_{(1,2)}$ Spec $^{\text {op }}$ based on the resolution $A_{\bullet} \rightarrow X$, by Theorem 8.7.

Notation 7.1 Given spectra $X$ and $Y$, let $\mathbf{E M}\{X, Y\}$ denote the topologically enriched subcategory of Spec consisting of all spectra in EM and mapping spaces between them, along with the objects $X$ and $Y$, with the mapping spaces $\operatorname{Spec}(X, A)$ and $\operatorname{Spec}(Y, A)$ for all $A$ in EM; c.f. [3, Remark 4.3], [6, Remark 7.5]. We consider the 2-track algebra $\Pi_{(1,2)} \mathbf{E M}\{X, Y\}^{\text {op }}$, or any 2 -track algebra $\mathcal{A}$ weakly equivalent to it. In the following construction, everything will take place within $\Pi_{(1,2)} \mathbf{E M}\{X, Y\}^{\text {op }}$, but we will write $\Pi_{(1,2)}$ Spec $^{\text {op }}$ for notational convenience.

Start with a class in the $E_{2}$-term:

$$
x \in E_{2}^{s, t}=\operatorname{Ext}_{\mathfrak{A}}^{s, t}\left(H^{*} X, H^{*} Y\right)=\operatorname{Ext}_{\mathfrak{A}}^{s, 0}\left(H^{*} X, \Sigma^{t} H^{*} Y\right)
$$

represented by a cocycle $x^{\prime}: F_{S} \rightarrow \Sigma^{t} H^{*} Y$, i.e., a map of $\mathfrak{A}$-modules satisfying $x^{\prime} d_{s}=0$. Realize $x^{\prime}$ as the cohomology of a map $x^{\prime \prime}: A_{s} \rightarrow \Sigma^{t} Y$ in Spec ${ }^{\text {op }}$. The equation $x^{\prime} d_{s}=0$ means that $x^{\prime \prime} d_{s}$ is null-homotopic; let $\gamma: x^{\prime \prime} d_{s} \rightarrow 0$ be a nullhomotopy. Consider the diagram in $\mathbf{S p e c}^{\mathrm{op}}$ :

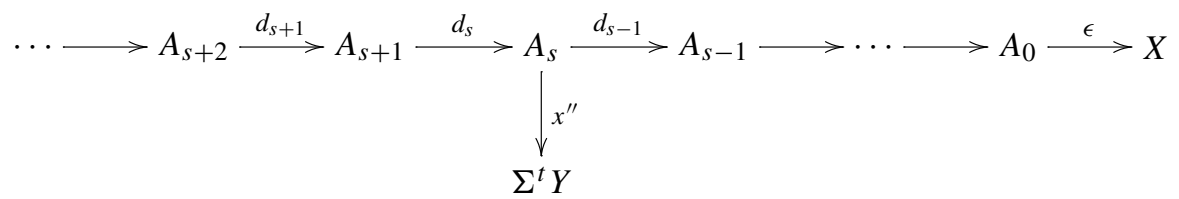


Now consider the underlying secondary pre-chain complex in $\Pi_{(1)}$ Spec $^{\text {op }}$ :

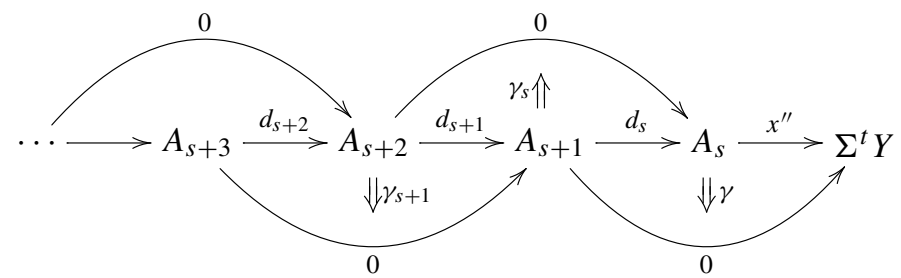

in which the obstructions $\mathcal{O}\left(\gamma_{i}, \gamma_{i+1}\right)$ are trivial, for $i \geq s$.

Theorem 7.2 The obstruction $\mathcal{O}\left(\gamma, \gamma_{s}\right) \in \pi_{1} \operatorname{Spec}^{\mathrm{op}}\left(A_{s+2}, \Sigma^{t} Y\right)=\pi_{0} \operatorname{Spec}^{\mathrm{op}}\left(A_{s+2}\right.$, $\left.\Sigma^{t+1} Y\right)$ is a (co)cycle and does not depend on the choices, up to (co)boundaries, and thus defines an element:

$$
d_{(2)}(x) \in \operatorname{Ext}_{\mathfrak{A}}^{s+2, t+1}\left(H^{*} X, H^{*} Y\right) .
$$

Moreover, this function

$$
d_{(2)}: \operatorname{Ext}_{\mathfrak{A}}^{s, t}\left(H^{*} X, H^{*} Y\right) \rightarrow \operatorname{Ext}_{\mathfrak{A}}^{s+2, t+1}\left(H^{*} X, H^{*} Y\right)
$$

is the Adams differential $d_{2}$.

Proof This is [3, Theorems 4.2 and 7.3], or the case $n=1, m=3$ of [6, Theorem 15.11].

Here we used the natural isomorphism:

$$
\operatorname{Ext}_{\pi_{0} \mathbf{E M}^{\mathrm{op}}}^{i, j}\left(H^{*} X, H^{*} Y\right) \cong \operatorname{Ext}_{\mathfrak{A}}^{i, j}\left(H^{*} X, H^{*} Y\right)
$$

where the left-hand side is defined as in Example 8.4. Using the equivalence of categories $H^{*}: \pi_{0} \mathbf{E M}^{\text {op }} \stackrel{\cong}{\rightarrow} \mathbf{M o d}_{\mathfrak{A}}^{\mathrm{fin}}$, this natural isomorphism follows from the natural isomorphisms:

$$
\begin{aligned}
\pi_{0} \operatorname{Spec}^{\mathrm{op}}\left(A_{s+2}, \Sigma^{t+1} Y\right) & =\operatorname{Hom}_{\mathfrak{A}}\left(F_{s+2}, H^{*} \Sigma^{t+1} Y\right) \\
& =\operatorname{Hom}_{\mathfrak{A}}\left(F_{s+2}, \Sigma^{t+1} H^{*} Y\right)
\end{aligned}
$$

Cocycles modulo coboundaries in this group are precisely $\operatorname{Ext}_{\mathfrak{A}}^{s+2, t+1}\left(H^{*} X, H^{*} Y\right)$.

Now assume that $d_{2}(x)=0$ holds, so that $x$ survives to the $E_{3}$-term. Since the obstruction

$$
\mathcal{O}\left(\gamma, \gamma_{s}\right)=\left(\gamma \otimes d_{s+1}\right) \square\left(x^{\prime \prime} \otimes \gamma_{s}\right)^{\boxminus}
$$


vanishes, one can choose a left 2-track $\xi: \gamma \otimes d_{s+1} \Rightarrow x^{\prime \prime} \otimes \gamma_{s}$, which makes (7.1) into a tertiary pre-chain complex in $\Pi_{(1,2)} \operatorname{Spec}^{\mathrm{op}}$. Since $(A, d, \gamma, \xi)$ was a tertiary chain complex to begin with, the obstructions $\mathcal{O}\left(\xi_{i}, \xi_{i+1}\right)$ are trivial, for $i \geq s$.

Theorem 7.3 The obstruction $\mathcal{O}\left(\xi, \xi_{s}\right) \in \pi_{2} \operatorname{Spec}^{\mathrm{op}}\left(A_{s+3}, \Sigma^{t} Y\right)=\pi_{0} \operatorname{Spec}^{\mathrm{op}}\left(A_{s+3}\right.$, $\left.\Sigma^{t+2} Y\right)$ is a (co)cycle and does not depend on the choices up to (co)boundaries, and thus defines an element:

$$
d_{(3)}(x) \in E_{3}^{s+3, t+2}(X, Y) \text {. }
$$

Moreover, this function

$$
d_{(3)}: E_{3}^{s, t}(X, Y) \rightarrow E_{3}^{s+3, t+2}(X, Y)
$$

is the Adams differential $d_{3}$.

Proof This is the case $n=2, m=4$ of [6, Theorem 15.11]. More precisely, by Theorem 9.3, the tertiary chain complex $(A, d, \gamma, \xi)$ in $\Pi_{(1,2)}$ Spec $^{\text {op }}$ yields a 2 nd order chain complex in $\mathrm{Nul}_{2} \mathbf{S p e c}^{\text {op }}$ based on the same EM-resolution $A_{\bullet} \rightarrow X$ in $\pi_{0}$ Spec $^{\mathrm{op}}$. The construction of $d_{(3)}$ above corresponds to the construction $d_{3}$ in [6, Definition 15.8].

Remark 7.4 The groups $E_{3}^{s, t}(X, Y)$ are an instance of the secondary Ext groups defined in [3, §4]. Likewise, the next term $E_{4}^{s, t}(X, Y)=\operatorname{ker} d_{(3)} / \operatorname{im} d_{(3)}$ is a higher order Ext group as defined in $[6, \S 15]$.

Theorem 7.5 A weak equivalence of 2-track algebras induces an isomorphism of higher Ext groups, compatible with the differential $d_{(3)}$. More precisely, let $F: \mathcal{A} \rightarrow$ $\mathcal{A}^{\prime}$ be a weak equivalence between 2-track algebras $\mathcal{A}$ and $\mathcal{A}^{\prime}$ which are weakly equivalent to $\Pi_{(1,2)} \mathbf{E M}\{X, Y\}^{\mathrm{op}}$. Then $F$ induces isomorphisms $E_{3, \mathcal{A}}^{s, t}(X, Y) \stackrel{\cong}{\cong}$ $E_{3, \mathcal{A}^{\prime}}^{s, t}(F X, F Y)$ making the diagram

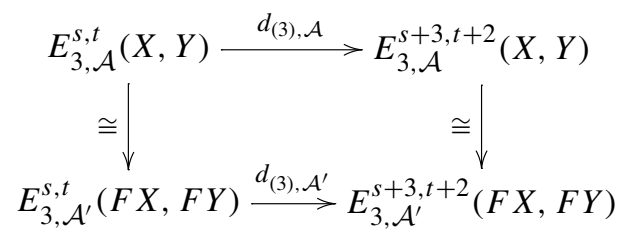

commute. Here the additional subscript $\mathcal{A}$ or $\mathcal{A}^{\prime}$ denotes the ambient 2-track category in which the secondary Ext groups and the differential are defined.

Proof This follows from the case $n=2$ of [6, Theorem 15.9], or an adaptation of the proof of [3, Theorem 5.1]. 


\section{Higher order resolutions}

In this section, we specialize some results of [6] about higher order resolutions to the case $n=2$. We use the fact that a 2-track algebra has an underlying algebra of left 2-cubical balls, which is the topic of Sect. 9.

First, we recall some background on relative homological algebra; more details can be found in $[3, \S 1]$.

Definition 8.1 Let $\mathbf{A}$ be an additive category and $\mathbf{a} \subseteq \mathbf{A}$ a full additive subcategory.

1. A chain complex $(A, d)$ is a -exact if for every object $X$ of a the chain complex $\operatorname{Hom}_{\mathbf{A}}\left(X, A_{\bullet}\right)$ is an exact sequence of abelian groups.

2. A chain map $f:(A, d) \rightarrow\left(A^{\prime}, d^{\prime}\right)$ is an a -equivalence if for every object $X$ of a, the chain map $\operatorname{Hom}_{\mathbf{A}}(X, f)$ is a quasi-isomorphism.

3. For an object $A$ of $\mathbf{A}$, an $A$-augmented chain complex $A_{\bullet}^{\epsilon}$ is a chain complex of the form

$$
\cdots \longrightarrow A_{1} \stackrel{d_{0}}{\longrightarrow} A_{0} \stackrel{\epsilon}{\longrightarrow} A \longrightarrow 0 \longrightarrow \cdots
$$

i.e., with $A_{-1}=A$ and $A_{n}=0$ for $n<-1$. Such a complex can be viewed as a chain map $\epsilon: A_{\bullet} \rightarrow A$ where $A$ is a chain complex concentrated in degree 0 . The map $\epsilon=d_{-1}$ is called the augmentation.

4. An a-resolution of $A$ is an $A$-augmented chain complex $A_{\bullet}^{\epsilon}$ which is a-exact and such that for all $n \geq 0$, the object $A_{n}$ belongs to a. In other words, an a-resolution of $A$ is a chain complex $A_{\bullet}$ in a together with an a-equivalence $\epsilon: A_{\bullet} \rightarrow A$.

Example 8.2 Consider the category $\mathbf{A}=\operatorname{Mod}_{R}$ of $R$-modules for some ring $R$, and the subcategory a of free (or projective) $R$-modules. This recovers the usual homological algebra of $R$-modules.

Definition 8.3 Let $\mathcal{A}$ be an abelian category and $F: \mathbf{A} \rightarrow \mathcal{A}$ an additive functor. The a -relative left derived functors of $F$ are the functors $L_{n}^{\mathbf{a}} F: \mathbf{A} \rightarrow \mathcal{A}$ for $n \geq 0$ defined by

$$
\left(L_{n}^{\mathbf{a}} F\right) A=H_{n}\left(F\left(A_{\bullet}\right)\right)
$$

where $A_{\bullet} \rightarrow A$ is any a-resolution of $A$.

Likewise, if $F: \mathbf{A}^{\mathrm{op}} \rightarrow \mathcal{A}$ is a contravariant additive functor, its a -relative right derived functors of $F$ are defined by

$$
\left(R_{\mathbf{a}}^{n} F\right) A=H^{n}\left(F\left(A_{\bullet}\right)\right) .
$$

Example 8.4 The a-relative Ext groups are given by

$$
\operatorname{Ext}_{\mathbf{a}}^{n}(A, B):=\left(R_{\mathbf{a}}^{n} \operatorname{Hom}_{\mathbf{A}}(-, B)\right)(A)=H^{n} \operatorname{Hom}_{\mathbf{A}}\left(A_{\bullet}, B\right) .
$$


Proposition 8.5 (Correction of 1-tracks) Let $\mathbf{B}$ be a category enriched in pointed groupoids, such that its homotopy category $\pi_{0} \mathbf{B}$ is additive. Let $\mathbf{a} \subseteq \pi_{0} \mathbf{B}$ be a full additive subcategory. Let $(A, d, \gamma)$ be a secondary pre-chain complex in $\mathbf{B}$ based on an $\mathbf{a}$-resolution $A_{\bullet} \rightarrow X$ of an object $X$ in $\pi_{0} \mathbf{B}$. Then there exists a secondary chain complex $\left(A, d, \gamma^{\prime}\right)$ in $\mathbf{B}$ with the same objects $A_{i}$ and differentials $d_{i}$. In particular $\left(A, d, \gamma^{\prime}\right)$ is also based on the a-resolution $A_{\bullet} \rightarrow X$.

Proof This follows from an adaptation of the proof of [3, Lemma 2.14], or the case $n=1$ of [6, Theorem 13.2].

Proposition 8.6 (Correction of 2-tracks) Let $\mathcal{A}$ be a 2-track algebra such that its homotopy category $\pi_{0} \mathcal{A}$ is additive. Let $\mathbf{a} \subseteq \pi_{0} \mathcal{A}$ be a full additive subcategory. Let $(A, d, \gamma, \xi)$ be a tertiary pre-chain complex in $\mathcal{A}$ based on an a-resolution $A_{\bullet} \rightarrow X$ of an object $X$ in $\pi_{0} \mathcal{A}$. Then there exists a tertiary chain complex $\left(A, d, \gamma, \xi^{\prime}\right)$ in $\mathcal{A}$ with the same objects $A_{i}$, differentials $d_{i}$, and left paths $\gamma_{i}$. In particular, $\left(A, d, \gamma, \xi^{\prime}\right)$ is also based on the a-resolution $A_{\bullet} \rightarrow X$.

Proof This follows from the case $n=2$ of [6, Theorem 13.2].

Theorem 8.7 (Resolution Theorem) Let $\mathcal{A}$ be a 2-track algebra such that its homotopy category $\pi_{0} \mathcal{A}$ is additive. Let $\mathbf{a} \subseteq \pi_{0} \mathcal{A}$ be a full additive subcategory. Let $A_{\bullet} \rightarrow X$ be an $\mathbf{a}$-resolution in $\pi_{0} \mathcal{A}$. Then there exists a tertiary chain complex in $\mathcal{A}$ based on the resolution $A_{\bullet} \rightarrow X$.

Proof This follows from the resolution theorems [6, Theorems 8.2 and 14.5].

\section{Algebras of left 2-cubical balls}

Proposition 9.1 Every left cubical ball of dimension 2 is equivalent to $C_{k}$ for some $k \geq 2$, where $C_{k}=B_{1} \cup \cdots \cup B_{k}$ is the left cubical ball of dimension 2 consisting of $k$ closed 2-cells going cyclically around the vertex 0 , with one common 1-cell $e_{i}$ between successive 2-cells $B_{i}$ and $B_{i+1}$, where by convention $B_{k+1}:=B_{1}$.

See Fig. 1, which is taken from [6, Figure 3].

Proof Let $B$ be a left cubical ball of dimension 2. For each closed 2-cell $B_{i}$, equipped with its homeomorphism $h_{i}: I^{2} \stackrel{\cong}{\rightarrow} B_{i}$, the faces $\partial_{1}^{1} B_{i}$ and $\partial_{2}^{1} B_{i}$ are required to be 1-cells of the boundary $\partial B \cong S^{1}$, while the faces $\partial_{1}^{0} B_{i}$ and $\partial_{2}^{0} B_{i}$ are not in $\partial B$, and therefore must be faces of some other 2-cells. In other words, we have $\partial_{1}^{0} B_{i}=\partial_{1}^{0} B_{j}$ or $\partial_{1}^{0} B_{i}=\partial_{2}^{0} B_{j}$ for some other 2-cell $B_{j}$, in fact a unique $B_{j}$, because $B$ is homeomorphic to a 2 -disk.

Pick any 2-cell of $B$ and call it $B_{1}$. Then the face $e_{1}:=\partial_{2}^{0} B_{1}$ appears as a face of exactly one other 2-cell, which we call $B_{2}$. The remaining face $e_{2}$ of $B_{2}$ appears as a face of exactly one other 2-cell, which we call $B_{3}$. Repeating this process, we list distinct 2-cells $B_{1}, \ldots, B_{k}$, and $B_{k+1}$ is one of the previously labeled 2-cells. Then $B_{k+1}$ must be $B_{1}$, with $e_{k}=\partial_{1}^{0} B_{1}$, since a 1-cell cannot appear as a common face of three 2-cells. Finally, this process exhausts all 2-cells, because all 2-cells share the common vertex 0 , which has a neighborhood homeomorphic to an open 2-disk. 

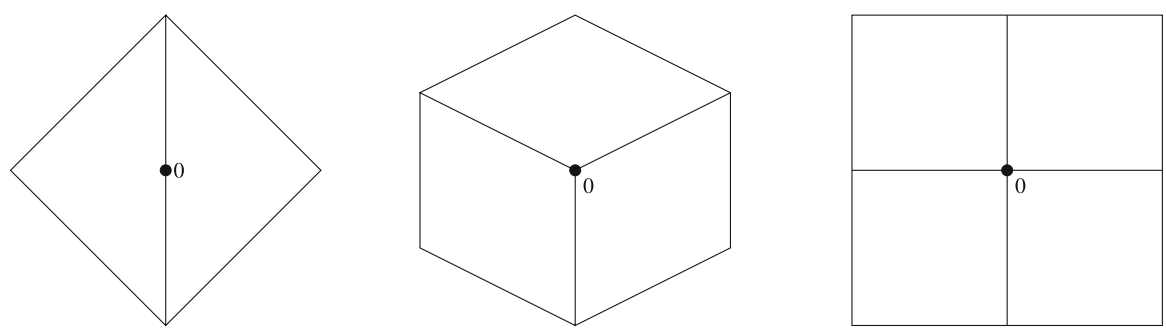

Fig. 1 The left cubical balls $C_{2}, C_{3}$, and $C_{4}$

Fig. 2 A left 2-cubical ball

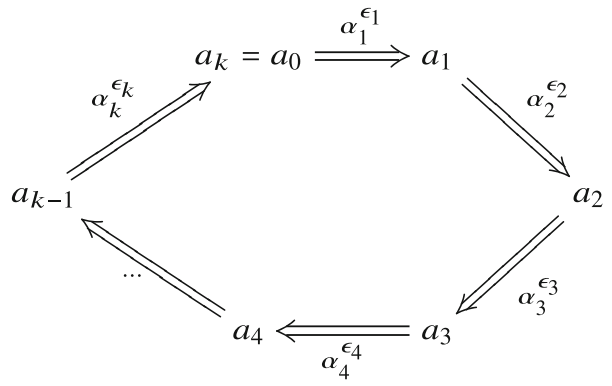

Proposition 9.2 A left 2-cubical ball [6, Definition 10.1] in a pointed space X corresponds to a circular chain of composable left 2-tracks:

$$
a=a_{0} \stackrel{\alpha_{1}^{\epsilon_{1}}}{\longrightarrow} a_{1} \stackrel{\alpha_{2}^{\epsilon_{2}}}{\longrightarrow} \cdots \rightarrow a_{k-1} \stackrel{\alpha_{k}^{\epsilon_{k}}}{\longrightarrow} a_{k}=a
$$

where the sign $\epsilon_{i}= \pm 1$ is the orientation of the 2-cells in the left cubical ball [6, Definition 10.8]. Moreover, such an expression $\left(\alpha_{1}, \ldots, \alpha_{k}\right)$ of a left 2-cubical ball is unique up to cyclic permutation of the $k$ left 2 -tracks $\alpha_{i}$. For example, $\left(\alpha_{1}, \alpha_{2}, \ldots, \alpha_{k}\right)$ and $\left(\alpha_{2}, \ldots, \alpha_{k}, \alpha_{1}\right)$ represent the same left 2-cubical ball. See Fig. 2.

Proof By our convention for the $\square$-composition, a left 2-track $\alpha$ defines a morphism between left paths $\alpha: d_{1}^{0} \alpha \Rightarrow d_{2}^{0} \alpha$. The gluing condition for a left 2-cubical ball $\left(\alpha_{1}, \ldots, \alpha_{k}\right)$ based on a left cubical ball $B=B_{1} \cup \cdots \cup B_{k}$ as in Proposition 9.1 is that the restrictions $\left.\alpha_{i}\right|_{e_{i}}$ and $\left.\alpha_{i+1}\right|_{e_{i}}$ agree on the common edge $e_{i} \subset B_{i} \cap B_{i+1}$. This is the composability condition for $\alpha_{i+1}^{\epsilon_{i+1}} \square \alpha_{i}^{\epsilon_{i}}$. Indeed, up to a global sign, the sign of $B_{i}$ is

$$
\epsilon_{i}= \begin{cases}+1 & \text { if } e_{i}=\partial_{2}^{0} B_{i} \\ -1 & \text { if } e_{i}=\partial_{1}^{0} B_{i}\end{cases}
$$

so that we have $\alpha_{i}^{\epsilon_{i}}:\left.\left.\alpha_{i}\right|_{e_{i-1}} \Rightarrow \alpha_{i}\right|_{e_{i}}$ and we may take $a_{i}=\left.\alpha_{i}\right|_{e_{i}}$.

Theorem 9.3 1. A 2-track algebra $\mathcal{A}$ yields an algebra of left 2-cubical balls $[6$, Definition 11.1] in the following way. Consider the system $\Theta(\mathcal{A}):=$ $\left(\left(\mathcal{A}_{(1,2)}, \otimes\right), \pi_{0} \mathcal{A}, D, \mathcal{O}\right)$, where: 
- $\left(\mathcal{A}_{(1,2)}, \otimes\right)$ is the underlying 2-graded category of $\mathcal{T}$ (described in Definition 5.1).

- $\pi_{0} \mathcal{A}$ is the homotopy category of $\mathcal{A}$.

- $q:(\mathcal{A})^{0}=\mathcal{A}_{(1) 0} \rightarrow \pi_{0} \mathcal{A}$ is the canonical quotient functor.

$-D:\left(\pi_{0} \mathcal{A}\right)^{\mathrm{op}} \times \pi_{0} \mathcal{A} \rightarrow \mathbf{A b}$ is the functor defined by $D(A, B)=$ $\pi_{2} \mathcal{A}_{(1,2)}(A, B)$.

- The obstruction operator $\mathcal{O}$ is obtained by concatenating the corresponding left 2-tracks and using the structural isomorphisms $\psi$ of the mapping 2-track groupoid:

$$
\begin{aligned}
\mathcal{O}_{B}\left(\alpha_{1}, \alpha_{2}, \ldots, \alpha_{k}\right) & =\psi_{a}\left(\alpha_{k}^{\epsilon_{k}} \square \cdots \square \alpha_{2}^{\epsilon_{2}} \square \alpha_{1}^{\epsilon_{1}}\right) \in \operatorname{Aut}_{\mathcal{A}_{(2)}(A, B)}(0) \\
& =\pi_{2} \mathcal{A}_{(1,2)}(A, B)
\end{aligned}
$$

where we denoted $a=\delta_{0} \alpha_{1}=\delta_{1} \alpha_{k}$.

2. Given a category $\mathcal{C}$ enriched in pointed spaces, $\Theta\left(\Pi_{(1,2)} \mathcal{C}\right)$ is the algebra of left 2-cubical balls

$$
\left(\mathrm{Nu}_{2} \mathcal{C}, \pi_{0} \mathcal{C}, \pi_{2} \mathcal{C}(-,-), \mathcal{O}\right)
$$

described in $[6, \S 11]$.

3. The construction $\Theta$ sends a tertiary pre-chain complex $(A, d, \delta, \xi)$ in $\mathcal{A}$ to a $2^{\text {nd }}$ order pre-chain complex in $\Theta(\mathcal{A})$, in the sense of [6, Definition 11.4]. Moreover, $(A, d, \delta, \xi)$ is a tertiary chain complex if and only if the corresponding $2^{\text {nd }}$ order pre-chain complex in $\Theta(\mathcal{A})$ is a $2^{\text {nd }}$ order chain complex.

Proof Let us check that the obstruction operator $\mathcal{O}$ is well-defined. By Proposition 9.2, the only ambiguity is the starting left 1 -cube $a_{i}$ in the composition. Two such compositions are conjugate in the groupoid $\mathcal{A}_{(2)}(A, B)$ :

$$
\begin{aligned}
& \alpha_{i-1}^{\epsilon_{i-1}} \square \cdots \square \alpha_{2}^{\epsilon_{2}} \square \alpha_{1}^{\epsilon_{1}} \square \alpha_{k}^{\epsilon_{k}} \square \cdots \square \alpha_{i+1}^{\epsilon_{i+1}} \square \alpha_{i}^{\epsilon_{i}} \\
& \quad=\left(\alpha_{i-1}^{\epsilon_{i-1}} \square \cdots \square \alpha_{1}^{\epsilon_{1}}\right) \square \alpha_{k}^{\epsilon_{k}} \square \cdots \square \alpha_{i+1}^{\epsilon_{i+1}} \square \alpha_{i}^{\epsilon_{i}} \square \cdots \square \alpha_{1}^{\epsilon_{1}} \square\left(\alpha_{i-1}^{\epsilon_{i-1}} \square \cdots \square \alpha_{1}^{\epsilon_{1}}\right)^{\boxminus} \\
& \quad=\beta^{\boxminus} \square \alpha_{k}^{\epsilon_{k}} \square \cdots \square \alpha_{1}^{\epsilon_{1}} \square \beta
\end{aligned}
$$

with $\beta=\left(\alpha_{i-1}^{\epsilon_{i-1}} \square \cdots \square \alpha_{1}^{\epsilon_{1}}\right)^{\boxminus}: a_{i} \Rightarrow a_{0}$. Since $\mathcal{A}_{(2)}(A, B)$ is a strictly abelian groupoid, we have the commutative diagram:

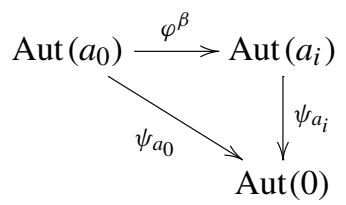

so that $\mathcal{O}_{B}\left(\alpha_{1}, \ldots, \alpha_{k}\right)$ is well-defined.

The remaining properties listed in [6, Definition 11.1] are straightforward verifications. 
Acknowledgements We thank the referee for their helpful comments. The second author thanks the MaxPlanck-Institut für Mathematik Bonn for its generous hospitality, as well as David Blanc, Robert Bruner, Dan Christensen, and Dan Isaksen for useful conversations.

\section{Appendix A: Models for homotopy 2-types}

Recall that the left $n$-cubical set $\operatorname{Nul}_{n}(X)$ of a pointed space $X$ depends only on the $n$-type $P_{n} X$ of $X[6, \S 1]$. In particular the fundamental 2-track groupoid $\Pi_{(1,2)}(X)$ depends only on the 2-type $P_{2} X$ of $X$. There are various algebraic models for homotopy 2-types in the literature, using 2-dimensional categorical structures. Let us mention the weak 2-groupoids of [15], the bigroupoids of [12], the double groupoids of [10], the two-typical double groupoids of [7], and the double groupoids with filling condition of [11].

In contrast, 2-track groupoids are not models for homotopy 2-types, not even of connected homotopy 2 -types. In the application we are pursuing, the functor $\Pi_{(1,2)}$ will be applied to topological abelian groups, hence products of Eilenberg-MacLane spaces. We are not trying to encode the homotopy 2-type of the Eilenberg-MacLane mapping theory, but rather as little information as needed in order to compute the Adams differential $d_{3}$.

The fundamental 2-track groupoid $\Pi_{(1,2)}(X)$ encodes the 1-type of $X$, via the fundamental groupoid $\Pi_{(1)}(X)$. Moreover, as noted in Remark 3.13, it also encodes the homotopy group $\pi_{2}(X)$. However, it fails to encode the $\pi_{1}(X)$-action on $\pi_{2}(X)$, as we will show below.

\section{A.1 Connected 2-track groupoids}

Recall that a category $\mathcal{C}$ is called skeletal if any isomorphic objects are equal. A skeleton of $\mathcal{C}$ is a full subcategory on a collection consisting of one representative object in each isomorphism class of objects of $\mathcal{C}$. Every groupoid is equivalent to a disjoint union of groups, that is, a coproduct of single-object groupoids. The inclusion sk $G \stackrel{\simeq}{\rightarrow} G$ of a skeleton of $G$ provides such an equivalence. A similar construction yields the following statement for 2-track groupoids.

Lemma A.1 Let $G=\left(G_{(1)}, G_{(2)}\right)$ be a 2-track groupoid.

1. There is a weak equivalence of 2-track groupoids $\operatorname{sk}_{(1)} G \stackrel{\sim}{\rightarrow} G$ where the first groupoid of $\mathrm{sk}_{(1)} G$ is skeletal.

2. If $G$ is connected and $G_{(1)}$ is skeletal, then there is a weak equivalence of 2-track groupoids $\operatorname{sk}_{(2)} G \stackrel{\sim}{\rightarrow} G$ where both groupoids of $\operatorname{sk}_{(2)} G$ are skeletal.

In particular, if $G$ is connected, then $\operatorname{sk}_{(2)} \operatorname{sk}_{(1)} G \stackrel{\sim}{\rightarrow} \operatorname{sk}_{(1)} G \stackrel{\sim}{\rightarrow} G$ is a weak equivalence between $G$ and a 2-track groupoid whose constituent groupoids are both skeletal.

Lemma A.2 Let $G$ and $G^{\prime}$ be connected 2-track groupoids whose constituent groupoids are skeletal. If there are isomorphisms of homotopy groups $\varphi_{1}: \pi_{1} G \simeq$ $\pi_{1} G^{\prime}$ and $\varphi_{2}: \pi_{2} G \simeq \pi_{2} G^{\prime}$, then there is a weak equivalence $\varphi: G \stackrel{\sim}{\rightarrow} G^{\prime}$. 
Proof Since $G_{(1)}$ and $G_{(1)}^{\prime}$ are skeletal, they are in fact groups, and the group isomorphism $\varphi_{1}$ is an isomorphism of groupoids $\varphi_{(1)}: G_{(1)} \stackrel{\simeq}{\rightarrow} G_{(1)}^{\prime}$.

Now we define a functor $\varphi_{(2)}: G_{(2)} \rightarrow G_{(2)}^{\prime}$. On objects, it is given by the composite

$$
\begin{aligned}
G_{(2) 0}=\operatorname{Comp} G_{(2)} \frac{q}{\simeq} & \operatorname{Star} G_{(1)}=G_{(1)}(0,0)=\pi_{1} G \stackrel{ }{\longrightarrow} \\
\frac{\varphi_{1}}{\simeq} & >\pi_{1} G^{\prime}=G_{(1)}^{\prime}(0,0)=\operatorname{Star} G_{(1)}^{\prime} \stackrel{q}{\simeq} \operatorname{Comp} G_{(2)}^{\prime}=G_{(2) 0}^{\prime}
\end{aligned}
$$

which is a bijection. On morphisms, $\varphi_{(2)}$ is defined as follows. We have $G_{(2)}(a, b)=\varnothing$ when $a \neq b$, so there is nothing to define then. On the automorphisms of an object $a \in G_{(2) 0}$, define $\varphi_{(2)}$ as the composite

$$
\begin{aligned}
G_{(2)}(a, a)= & \operatorname{Aut}_{G_{(2)}}(a) \stackrel{\psi_{a}}{\simeq} \operatorname{Aut}_{G_{(2)}}(0)=\pi_{2} G \longrightarrow \\
& \underset{\varphi_{2}}{\simeq} \pi_{2} G^{\prime}=\operatorname{Aut}_{G_{(2)}^{\prime}}\left(0^{\prime}\right) \underset{\psi_{\varphi(a)}^{\prime}}{\simeq} \operatorname{Aut}_{G_{(2)}^{\prime}}(\varphi(a))=G_{(2)}^{\prime}(\varphi(a), \varphi(a)) .
\end{aligned}
$$

Then $\varphi_{(2)}$ is a functor and commutes with the structural isomorphisms, by construction. Thus $\varphi=\left(\varphi_{(1)}, \varphi_{(2)}\right): G \rightarrow G^{\prime}$ is a morphism of 2-track groupoids, and is moreover a weak equivalence.

Corollary A.3 Let $G$ and $G^{\prime}$ be connected 2-track groupoids with isomorphic homotopy groups $\pi_{i} G \simeq \pi_{i} G^{\prime}$ for $i=1,2$. Then $G$ and $G^{\prime}$ are weakly equivalent, i.e., there is a zigzag of weak equivalences between them.

Proof Consider the zigzag of weak equivalences

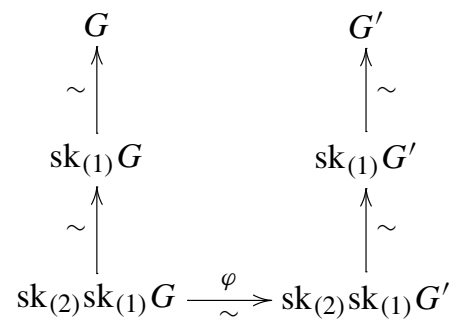

where the bottom morphism $\varphi$ is obtained from Lemma A.2.

By Remark 3.13, the functor $\Pi_{(1,2)}: \mathbf{T o p}_{*} \rightarrow \mathbf{G p d}_{(\mathbf{1}, \mathbf{2})}$ induces a functor

$$
\Pi_{(1,2)}: \text { Ho (connected 2-Types) } \rightarrow \text { Ho }\left(\operatorname{Gpd}_{(\mathbf{1}, 2)}\right)
$$

where the left-hand side denotes the homotopy category of connected 2-types (localized with respect to weak homotopy equivalences), and the right-hand side denotes the localization with respect to weak equivalences, as in Definition 3.12. 
Proposition A.4 The functor $\Pi_{(1,2)}$ in (A.1) is not an equivalence of categories.

Proof Let $X$ and $Y$ be connected 2-types with isomorphic homotopy groups $\pi_{1}$ and $\pi_{2}$, but distinct $\pi_{1}$-actions on $\pi_{2}$. Then $X$ and $Y$ are not weakly equivalent, but $\Pi_{(1,2)}(X)$ and $\Pi_{(1,2)}(Y)$ are weakly equivalent, by Corollary A.3.

\section{A.2 Comparison to bigroupoids}

Any algebraic model for (pointed) homotopy 2-types has an underlying 2-track groupoid. Using the globular description in Remark 3.1, the most direct comparison is to the bigroupoids of [12]. A pointed bigroupoid (resp. double groupoid) will mean one equipped with a chosen object, here denoted $x_{0}$ to emphasize that it unrelated to the algebraic structure of the bigroupoid.

Proposition A.5 Let $\Pi_{2}^{\mathrm{BiGpd}}(X)$ denote the homotopy bigroupoid of a space $X$ constructed in [12], where it was denoted $\Pi_{2}(X)$.

1. There is a forgetful functor $U$ from pointed bigroupoids to 2-track groupoids.

2. For a pointed space $X$, there is a natural isomorphism of 2-track groupoids $\Pi_{(1,2)}(X) \cong U \Pi_{2}^{\mathrm{BiGpd}}(X)$.

Proof Let $B$ be a bigroupoid. We construct a 2-track groupoid $U B$ as follows. The first constituent groupoid of $U B$ is the underlying groupoid of $B$

$$
U B_{(1)}:=\pi_{0} B
$$

obtained by taking the components of each mapping groupoid $B(x, y)$. The second constituent groupoid of $U B$ is a coproduct of mapping groupoids

$$
U B_{(2)}:=\coprod_{x \in \operatorname{Ob}(B)} B\left(x, x_{0}\right) .
$$

The quotient function $q: U B_{(2) 0} \rightarrow \operatorname{Star} U B_{(1)}$ is induced by the natural quotient maps $\mathrm{Ob}\left(B\left(x, x_{0}\right)\right) \rightarrow \pi_{0} B\left(x, x_{0}\right)$. To define the structural isomorphisms

$$
\psi_{a}: \operatorname{Aut}(a) \stackrel{\cong}{\rightarrow} \operatorname{Aut}\left(c_{x_{0}}\right)
$$

for objects $a \in U B_{(2) 0}$, which are 1-morphisms to the basepoint $a: x \rightarrow x_{0}$, consider the diagram 


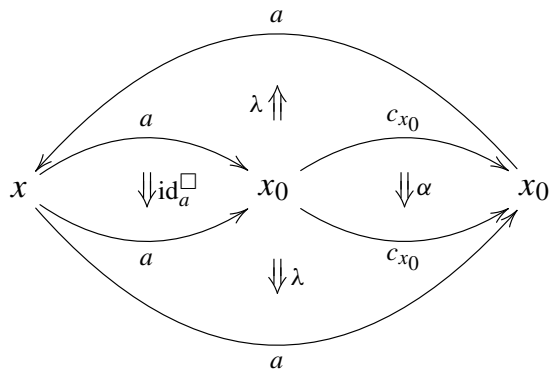

where $\lambda: c_{x_{0}} \bullet a \Rightarrow a$ is the left identity coherence 2 -isomorphism, $\bullet$ denotes composition of 1-morphisms, and $c_{x_{0}}$ is the identity 1-morphism of the object $x_{0}$. (We kept our notation $\square$ for composition of 2-morphisms.) The inverse $\psi_{a}^{-1}: \operatorname{Aut}\left(c_{x_{0}}\right) \rightarrow \operatorname{Aut}(a)$ is defined by going from top to bottom in the diagram, namely

$$
\psi_{a}^{-1}(\alpha)=\lambda \square\left(\alpha \bullet \mathrm{id}_{a}^{\square}\right) \square \lambda^{\boxminus}
$$

One readily checks that $U B$ is a 2-track groupoid, that this construction $U$ is functorial, and that $U \Pi_{2}^{\mathrm{BiGpd}}(X)$ is naturally isomorphic to $\Pi_{(1,2)}(X)$ as 2-track groupoids.

\section{A.3 Comparison to double groupoids}

The homotopy double groupoid $\rho_{2}^{\square}(X)$ from [10] is a cubical construction. Following the terminology therein, double groupoid will be shorthand for edge symmetric double groupoid with connection.

Let us recall the geometric idea behind $\rho_{2}^{\square}(X)$. A path $a: I \rightarrow X$ has an underlying semitrack $\langle a\rangle$, defined as its equivalence class with respect to thin homotopy rel $\partial I$. A semitrack $\langle a\rangle$ in turn has an underlying track $\{a\}$. A square $u: I^{2} \rightarrow X$ has an underlying 2-track $\{u\}$. A 2-track $\{u\}$ in turn has an underlying equivalence class $\{u\}_{T}$ with respect to cubically thin homotopy, i.e., a homotopy whose restriction to the boundary $\partial I^{2}$ is thin (not necessarily stationary). The homotopy double groupoid $\rho_{2}^{\square}(X)$ encodes semitracks $\langle a\rangle$ in $X$ and 2-tracks $\{u\}_{T}$ up to cubically thin homotopy.

Proposition A.6 Let $\rho_{2}^{\square}(X)$ denote the homotopy double groupoid of a space $X$ constructed in [10].

1. There is a forgetful functor $U$ from pointed double groupoids to 2-track groupoids.

2. For a pointed space $X$, there is a natural weak equivalence of 2-track groupoids $\Pi_{(1,2)}(X) \stackrel{\sim}{\rightarrow} U \rho_{2}^{\square}(X)$. 
Proof We adopt the notation of [10], including that compositions in a double groupoid are written in diagrammatic order, i.e., $a+b$ denotes the composition $x \stackrel{a}{\rightarrow} y \stackrel{b}{\rightarrow} z$. However, we keep our graphical convention for the two axes:

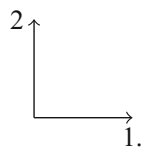

Let $D$ be a double groupoid, whose data is represented in the diagram of sets

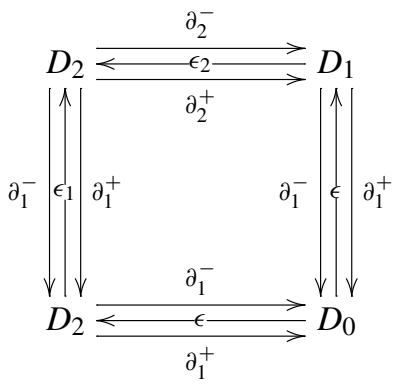

along with connections $\Gamma^{-}, \Gamma^{+}: D_{1} \rightarrow D_{2}$. Two 1-morphisms $a, b \in D_{1}$ with same endpoints $\partial_{1}^{-}(a)=\partial_{1}^{-}(b)=x, \partial_{1}^{+}(a)=\partial_{1}^{+}(b)=y$ are called homotopic if there exists a 2-morphism $u \in D_{2}$ satisfying $\partial_{2}^{-}(u)=a, \partial_{2}^{+}(u)=b, \partial_{1}^{-}(u)=\epsilon(x)$, $\partial_{2}^{+}(u)=\epsilon(y)$. We write $a \sim b$ if $a$ and $b$ are homotopic.

We now define the underlying 2-track groupoid $U D$. The first constituent groupoid $U D_{(1)}$ has object set $D_{0}$ and morphism set $D_{1} / \sim$, with groupoid structure inherited from the groupoid $\left(D_{0}, D_{1}\right)$. The second constituent groupoid $U D_{(2)}$ has object set

$$
U D_{(2) 0}:=\left\{a \in D_{1} \mid \partial_{1}^{+}(a)=x_{0}\right\}
$$

A morphism in $U D_{(2)}$ from $a$ to $b$ is an element $u \in D_{2}$ satisfying $\partial_{1}^{-}(u)=a$, $\partial_{2}^{-}(u)=b, \partial_{1}^{+}(u)=\epsilon\left(x_{0}\right), \partial_{2}^{+}(u)=\epsilon\left(x_{0}\right)$, as illustrated here:

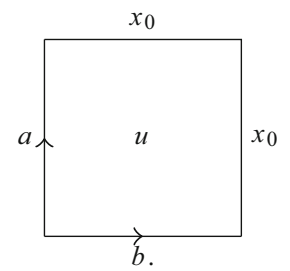

Composition in $U D_{(2)}$ is defined as follows. Given 1-morphisms $a, b, c: x \rightarrow x_{0}$ in $D_{1}$ and morphisms $u: a \Rightarrow b$ and $v: b \Rightarrow c$ in $U D_{(2)}$, their composition $v \square u: a \Rightarrow c$ is defined by 


$$
\begin{aligned}
v \square u & =\left(\Gamma^{+}(b)+2 u\right)+{ }_{1}\left(v+2 \odot_{x_{0}}\right) \\
& =\left(\Gamma^{+}(b)+2 u\right)+{ }_{1} v \\
& =\left(\Gamma^{+}(b)+1 v\right)+2 u
\end{aligned}
$$

as illustrated here:

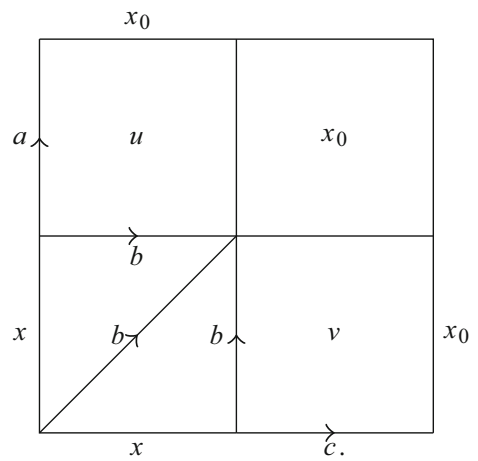

The identity morphisms in $U D_{(2)}$ are given by $\mathrm{id}_{a}^{\square}=\Gamma^{-}(a)$. The inverse of $u: a \Rightarrow b$ is given by

$$
\begin{aligned}
u^{\boxminus} & =\left(\left(--_{1}\right) \Gamma^{+}(b)+{ }_{2}\left(--_{1}\right) u\right)+{ }_{1}\left(\epsilon_{2}(a)+{ }_{2} \Gamma^{-}(a)\right) \\
& =\left(\left(--_{1}\right) \Gamma^{+}(b)+2(-1) u\right)+{ }_{1} \Gamma^{-}(a)
\end{aligned}
$$

as illustrated here:

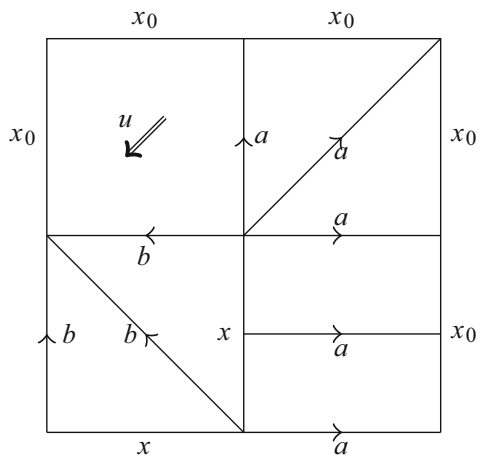

The structural isomorphisms $\psi_{a}^{-1}: \operatorname{Aut}\left(\epsilon\left(x_{0}\right)\right) \rightarrow \operatorname{Aut}(a)$ are defined by

$$
\begin{aligned}
\psi_{a}^{-1}(u) & =\left(\Gamma^{-}(a)+{ }_{2} \odot_{x_{0}}\right)+{ }_{1}\left(\odot_{x_{0}}+2 u\right) \\
& =\Gamma^{-}(a)+{ }_{1} u \\
& =\Gamma^{-}(a)+{ }_{2} u
\end{aligned}
$$


as illustrated here:

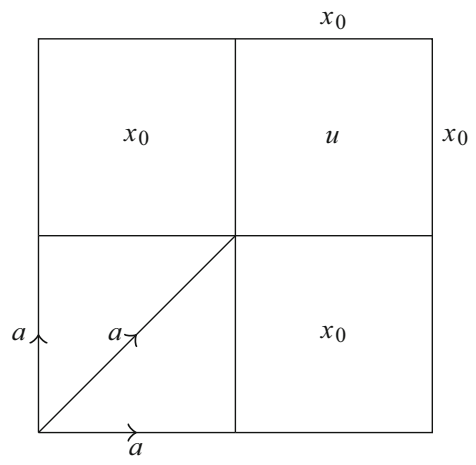

The quotient function $q: U D_{(2) 0} \rightarrow$ Star $U D_{(1)}$ is induced by the quotient function $D_{1} \rightarrow D_{1} / \sim$. One readily checks that $U D$ is a 2 -track groupoid, and that this construction $U$ is functorial.

For a pointed space $X$, define a comparison map $\Pi_{(1,2)}(X) \rightarrow U \rho_{2}^{\square}(X)$ which is an isomorphism on $\Pi_{(1)}(X)$, and which quotients out the thin homotopy relation between left paths in $X$ and the cubically thin homotopy relation between left 2-tracks. This defines a natural weak equivalence of 2-track groupoids.

\section{References}

1. Adams, J.F.: On the structure and applications of the Steenrod algebra. Comment. Math. Helv. 32, 180-214 (1958)

2. Baues, H.-J.: The Algebra of Secondary Cohomology Operations, Progress in Mathematics, vol. 247. Birkhäuser Verlag, Basel (2006)

3. Baues, H.-J., Jibladze, M.: Secondary derived functors and the Adams spectral sequence. Topology 45(2), 295-324 (2006). doi:10.1016/j.top.2005.08.001

4. Baues, H.J., Blanc, D.: Stems and spectral sequences. Algebr. Geom. Topol. 10(4), 2061-2078 (2010). doi:10.2140/agt.2010.10.2061

5. Baues, H.-J., Jibladze, M.: Dualization of the Hopf algebra of secondary cohomology operations and the Adams spectral sequence. J. K-Theory 7(2), 203-347 (2011). doi:10.1017/is010010029jkt133

6. Baues, H.-J., Blanc, D.: Higher order derived functors and the Adams spectral sequence. J. Pure Appl. Algebra 219(2), 199-239 (2015). doi:10.1016/j.jpaa.2014.04.018

7. Blanc, D., Paoli, S.: Two-track categories. J. K-Theory 8(1), 59-106 (2011). doi:10.1017/ is010003020jkt116

8. Bousfield, A.K., Kan, D.M.: The homotopy spectral sequence of a space with coefficients in a ring. Topology 11, 79-106 (1972)

9. Bousfield, A.K., Friedlander, E.M.: Homotopy theory of a spaces, spectra, and bisimplicial sets, Geometric applications of homotopy theory (Proc. Conf., Evanston, Ill., 1977): II, Lecture Notes in Math., vol. 658, pp. 80-130. Springer, Berlin (1978)

10. Brown, R., Hardie, K.A., Kamps, K.H., Porter, T.: A homotopy double groupoid of a Hausdorff space. Theory Appl. Categ. 10, 71-93 (2002)

11. Cegarra, A.M., Heredia, B.A., Remedios, J.: Double groupoids and homotopy 2-types. Appl. Categ. Struct. 20(4), 323-378 (2012). doi:10.1007/s10485-010-9240-1

12. Hardie, K.A., Kamps, K.H., Kieboom, R.W.: A homotopy bigroupoid of a topological space. Appl. Categ. Struct. 9(3), 311-327 (2001). doi:10.1023/A:1011270417127

13. Mandell, M.A., May, J.P., Schwede, S., Shipley, B.: Model categories of diagram spectra. Proc. Lond. Math. Soc. (3) 82(2), 441-512 (2001). doi:10.1112/S0024611501012692 
14. Maunder, C.R.F.: On the differentials in the Adams spectral sequence. Proc. Camb. Philos. Soc. 60, 409-420 (1964)

15. Tamsamani, Z.: Sur des notions de n-catégorie et n-groupoïde non strictes via des ensembles multisimpliciaux. K-Theory 16(1), 51-99 (1999). doi:10.1023/A:1007747915317 (French, with English summary) 